Check for updates

Cite this: Phys. Chem. Chem. Phys., 2021, 23, 26602

Received 30th August 2021, Accepted 8th November 2021 DOI: $10.1039 / \mathrm{d} 1 \mathrm{cp} 03975 \mathrm{j}$

rsc.li/pccp

\title{
Rotational action spectroscopy of trapped molecular ions
}

\author{
Oskar Asvany (D)* and Stephan Schlemmer (D)
}

\begin{abstract}
Rotational action spectroscopy is an experimental method in which rotational spectra of molecules, typically in the microwave to sub-mm-wave domain of the electromagnetic spectrum $(\sim 1-1000 \mathrm{GHz}$ ), are recorded by action spectroscopy. Action spectroscopy means that the spectrum is recorded not by detecting the absorption of light by the molecules, but by the action of the light on the molecules, e.g., photon-induced dissociation of a chemical bond, a photon-triggered reaction, or photodetachment of an electron. Typically, such experiments are performed on molecular ions, which can be well controlled and mass-selected by guiding and storage techniques. Though coming with many advantages, the application of action schemes to rotational spectroscopy was hampered for a long time by the small energy content of a corresponding photon. Therefore, the first rotational action spectroscopic methods emerged only about one decade ago. Today, there exists a toolbox full of different rotational action spectroscopic schemes which are summarized in this review.
\end{abstract}

\section{Introduction}

Among the different spectroscopy regimes, rotational spectroscopy takes a prominent role, as it permits an unperturbed determination of the molecular structure, has high sensitivity, and because it probes the lowest molecular quantum levels, typically in the microwave to sub-mm-wave domain of the electromagnetic spectrum $(\sim 1-1000 \mathrm{GHz})$. Because of the latter fact, molecules in cold interstellar clouds can be detected in emission with high sensitivity, without the need for bright background sources. Since the first radio astronomical detection of a molecule in space, the $\Lambda$-type doublet of $\mathrm{OH}$ at

I. Physikalisches Institut, Universität zu Köln, Zülpicher Str. 77, 50937 Köln, Germany. E-mail: asvany@ph1.uni-koeln.de
1.6 GHz, in $1963,{ }^{1}$ microwave and later (sub)mm-wave spectroscopic techniques thus dominated the astronomical detection of molecules, of which more than 220 are now known to exist in the interstellar medium or circumstellar shells. ${ }^{2,3}$ Closely connected with these developments are also the laboratory rotational spectroscopy techniques, examples being the standard absorption cell, the Fourier-transform microwave (FT-MW) method developed by Balle and Flygare in the 1980s, ${ }^{4}$ and, more recently, the chirped pulse Fourier-transform technique (CP-FT, ref. 5).

Another important subset of spectroscopy concerns the spectroscopy of molecular ions, and a huge body of rotational work has already been accumulated by the techniques mentioned above, see e.g. ref. 6-18. Ions take a prominent role in

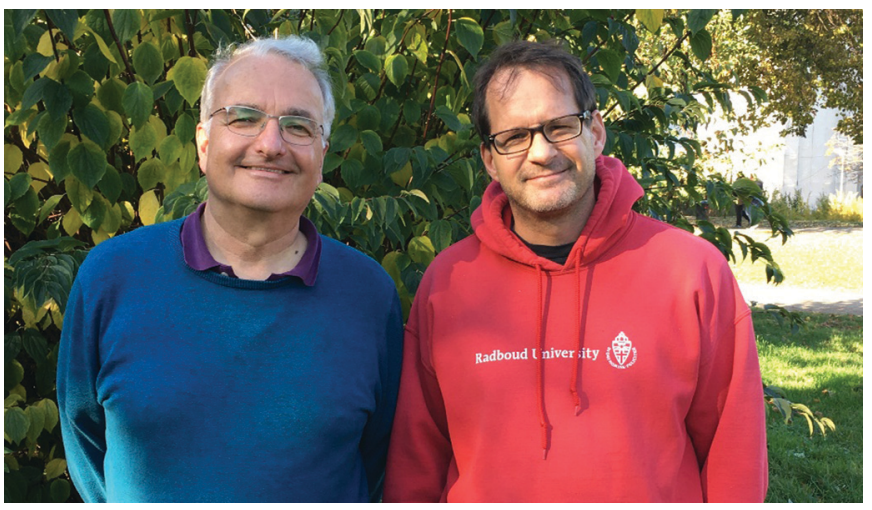

Stephan Schlemmer and Oskar Asvany
Stephan Schlemmer obtained his PhD in Göttingen 1991, and after working as PostDoc in Berkeley, he finished his Habilitation 2001 in Chemnitz. He heads the spectroscopy group at the I. Physikalisches Institut at the Universität zu Köln. His research interests are molecular physics and spectroscopy, in particular floppy molecular systems. Oskar Asvany studied physics in Bonn, Lund, and Taipei, and received his PhD 2003 in Chemnitz. Since 2006 he works at the Universität zu Köln, concentrating on action spectroscopy in ion traps. He finished his Habilitation in 2021. 
astrochemistry, ${ }^{19-22}$ as they are formed readily in space by ionizing radiation and cosmic rays, and because they can substantially influence interstellar chemistry by fast ion-molecule reactions at temperatures as low as $10 \mathrm{~K}$. This efficiency can be traced back to the high collision rate due to the attraction between a charge and an induced dipole, and because of the typically low energy barriers of such reactions. Traditionally, ions are generated igniting a discharge of a suitable neutral precursor gas mixture in a cooled absorption cell or at the nozzle tip of a pulsed supersonic expansion. Unfortunately, the discharge produces a variety of different species (ions, excited neutrals, radicals), which is particularly severe for hydrocarbon molecules, and the missing selection between them poses a severe problem. A famous example for this difficulty is Takeshi Oka's quest for the IR spectrum of $\mathrm{CH}_{5}{ }^{+},{ }^{23}$ the 'enfant terrible' of molecular spectroscopy, ${ }^{24}$ using a discharge through a $\mathrm{CH}_{4}-\mathrm{H}_{2}-\mathrm{He}$ mixture, for which he needed decades to distinguish the spectroscopic signals of the ions $\mathrm{CH}_{5}{ }^{+}, \mathrm{CH}_{4}{ }^{+}, \mathrm{CH}_{3}{ }^{+}$, etc. and even of excited methane, $\mathrm{CH}_{4}^{*}$.

The problem named above can be avoided by using ion trap setups. These offer the advantage of cryogenic cooling and mass selection, leading to simple and uncontaminated spectra. In addition, the stored ions can be exposed to the radiation field for rather long times, and, therefore, very high spectral resolution can be achieved in principle. The limited number of trapped ions (in the range $10^{3}$ to $10^{6}$ ), though, makes the application of some sort of action spectroscopy mandatory. In this approach the action of the photons on the trapped ionic molecules is detected by subsequent mass selection and counting of parent or product/fragment ions. As counting of the ions has very high efficiency, action spectroscopy is furthermore very sensitive. In the realm of IR laser spectroscopy of molecular ions, action schemes have been routinely applied since the 1980s for vibrational or even electronic investigations with methods like predissociation spectroscopy, ${ }^{25-28}$ infrared multiphoton dissociation (IRMPD), ${ }^{29-31}$ or the messenger (tagging) technique. ${ }^{32-36}$ Later, the toolbox of IR action spectroscopy was enriched with methods like laser induced reactions (LIR), ${ }^{37-40}$ and laser induced inhibition of complex growth (LIICG), ${ }^{4-44}$ which are presented in more detail in the following sections.

In view of the above-mentioned importance of $\mathrm{mm}$-wave and submm-wave (THz) spectroscopy to astronomy, it was desirable to transfer the advantages of action spectroscopy to rotational spectroscopy of molecular ions. However, the inherent low photon energy has hampered the development of such rotational methods for a long time. The first demonstration of pure rotational spectroscopy in a cold ion trap was a direct LIR process such as $\mathrm{H}_{2} \mathrm{D}^{+}+\mathrm{H}_{2}+h \nu \rightarrow \mathrm{H}_{3}{ }^{+}+\mathrm{HD}$, by which the fundamental transitions $1_{01} \leftarrow 0_{00}$ and $1_{11} \leftarrow 0_{00}$ of $p-\mathrm{H}_{2} \mathrm{D}^{+}$and $o-\mathrm{D}_{2} \mathrm{H}^{+}$, respectively, could be recorded in the $\mathrm{THz}$ regime. ${ }^{45}$ But this first laboratory demonstration was only possible due to fortuitous chemical and physical circumstances, namely the large rotational spacing of the light ions $\mathrm{H}_{2} \mathrm{D}^{+}$and $\mathrm{D}_{2} \mathrm{H}^{+}$, and also due to the existence of a reaction with a low energy barrier (which had to be surmounted by the photon energy). Such a scheme is thus unique and cannot be transferred to other molecular ions in a direct manner. Thus, other, more general rotational action schemes had to be devised.

This work presents a review of novel rotational action spectroscopic methods developed in the last decade. The requirements on the cryogenic trap machines and optics is briefly summarized in Section 2, and some background on vibrational and electronic action spectroscopy is given in Section 3. The developed rotational action schemes are summarized and discussed in detail in Section 4, with an emphasis on the double-resonance techniques developed in Cologne. A particularly exciting recent development is the high-precision rotational spectroscopy of sympathetically cooled ions. Due to its different technical character, it is treated separately in Section 5. An outlook is given in Section 6.

\section{Experimental prerequisites}

The general principles of an action spectroscopy experiment are explained with the help of Fig. 1. Typically, such a setup consists of an ion source, a first mass filter (which could be a quadrupole or a time-of-flight mass analyzer), the ion trap, followed again by a second mass filter and finally the ion detector. Several such cryogenic trap machines have been described in the literature in detail, ${ }^{35,42,46-53}$ as well as the central part, typically a multipolar cryogenic ion trap. ${ }^{54-56}$ The experiments are conducted in a pulsed mode. The pulse of ions generated in the ion source is mass filtered for the ionic species to be investigated in the first filter and then injected into the ion trap. Here the ions can be stored and cooled. The cooling of the translational and internal degrees of freedom usually happens by inelastic collisions with a cold buffer gas, mostly by (a pulse of) helium. In the trap, the ions are excited by narrow-bandwidth and tunable radiation ((sub)mm-wave, IR, vis/UV). For the double-resonance rotational experiments described in Section 4, the (sub)mm-wave and IR beams have to be combined and applied at the same time. For action spectroscopy to work, this excitation has to change the mass composition of the trapped ion ensemble, be it by promoting or hindering a bimolecular reaction, or by simply dissociating or neutralizing the molecular ions. This change of mass composition can be monitored by releasing the total trap content into the second mass filter and counting the mass-filtered ionic reaction products (or fragments) in the detector.

For rotational action spectroscopy, one has to adapt such a machine to the applied long wavelength radiation. As radiation at $30 \mathrm{GHz}$, for instance, has a wavelength of $1 \mathrm{~cm}$, it is getting increasingly cumbersome to refocus the divergent invisible

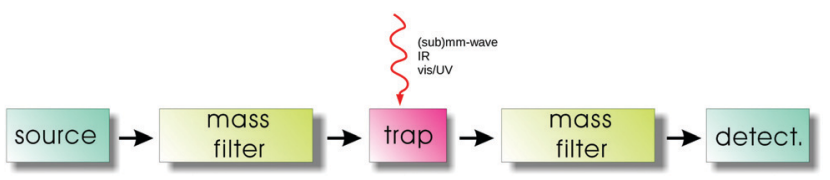

Fig. 1 Schematic setup of an action spectroscopy trap experiment. 
beam through long distances along the axis of the machine, particularly through small orifices. A practical approach is therefore to have a bent machine setup, with a quadrupole ion bender inserted between the first mass filter and the trap (such a bender is not shown in Fig. 1), such to allow for a close proximity of the microwave/mm-wave source to the trap. An alternative possibility is an ion trap with a lateral irradiation window as recently designed by the Innsbruck group. ${ }^{56}$

Another aspect concerns the vacuum window material for the long wavelength radiation. The most important criteria for this choice are the ultrahigh vacuum compatibility and transparency for the microwave to submillimeter wave region (10-1500 GHz). Apart from this region, double resonance experiments require transparency also in the IR, and future double resonance with electronic excitation potentially even need transparency in the visible and UV regions. Typical window and optical materials used in the mm-wave community, like high density polyethylene (HDPE), polytetrafluorethylene (PTFE), polymethylpentene (TPX) or Teflon do not fulfill the latter requirements. In addition, they exhibit bad vacuum performance, be it because of permeability of air and moisture or because of severe outgassing due to porousness. Window materials suitable for the application here are either $z$-cut quartz or chemical vapour deposition (CVD) diamond. In particular CVD-diamond, though expensive and with a high refractive index $(n \approx 2.38$ ), is transparent from the near UV to far into the microwave region and is a very robust material.

\section{Action spectroscopy}

\subsection{Why action spectroscopy?}

In contrast to conventional absorption spectroscopy, where one detects what the molecules do to the light, in action spectroscopy one detects what the light is doing to the molecules in case of absorption. This action spectroscopy is best done with ions, as their charge offers a firm handle to control, i.e. guide, mass select, trap, and finally count them. As this counting can be done with an efficiency close to unity (e.g. with a Daly detector ${ }^{57}$ ), action spectroscopy is very sensitive (while counting of photons close to the quantum limit can be achieved only in exceptional cases). For example, action spectroscopy can operate with a few hundred stored ions (see $e . g$. the ordinate of Fig. 8) in a trap volume of about $3 \mathrm{~cm}^{3}$ (see e.g. ref. 55) and thus an ion density on the order of $100 \mathrm{~cm}^{-3}$, whereas typical absorption experiments require a minimum number density of the investigated species on the order of $10^{8} \mathrm{~cm}^{-3}$. Other advantages of action spectroscopy are (i) the mass selection, which is often done with compact quadrupole mass selectors, (ii) the cooling capability in cryogenic ion traps, leading to narrow Doppler widths and favorable partition functions (important for complex and floppy molecules), (iii) long interaction times (typically up to several seconds), and finally (iv) the consumption of only tiny amounts of the precursor sample. The latter feature is especially important when one makes experiments with expensive (e.g. isotopically substituted), poisonous or explosive precursors.

Action spectroscopic methods have been applied since the 1980s, mainly for rovibrational and electronic spectroscopy of ionic molecules and complexes. Because rotational action spectroscopy is based on these developments, they are briefly reviewed here, with a focus on the methods LIR and LIICG.

\subsection{Early days of action spectroscopy}

One of the first experiments in which ion detection is used to record spectra of molecular ions is the Doppler-tuned fast-ionbeam laser spectrometer constructed by Wing and coworkers in the 1970s in Tucson, Arizona. It is depicted in Fig. 2. It uses the acceleration of an ion beam (several $\mathrm{kV}$ ) to Doppler-tune transitions of the molecular ions through a fixed-frequency (but otherwise stepwise tunable) high-power CO-laser. The ions are mass-analyzed, and the excitation of the molecular ions is detected via their different interaction behavior (neutralization, dissociation, scattering) in a gas target and subsequent recording of the ion current in a Faraday cup (see Fig. 2). Many fundamental molecular ions have been measured in the IR for the first time with this setup, as $\mathrm{HD}^{+},{ }^{58} \mathrm{HeH}^{+},{ }^{59} \mathrm{D}_{3}{ }^{+60}$ and $\mathrm{H}_{2} \mathrm{D}^{+} .{ }^{61}$ Although pioneering, the disadvantages of this method are that ions are not cooled and thus produced with internal excitation, the short interaction times, and the insensitivity of

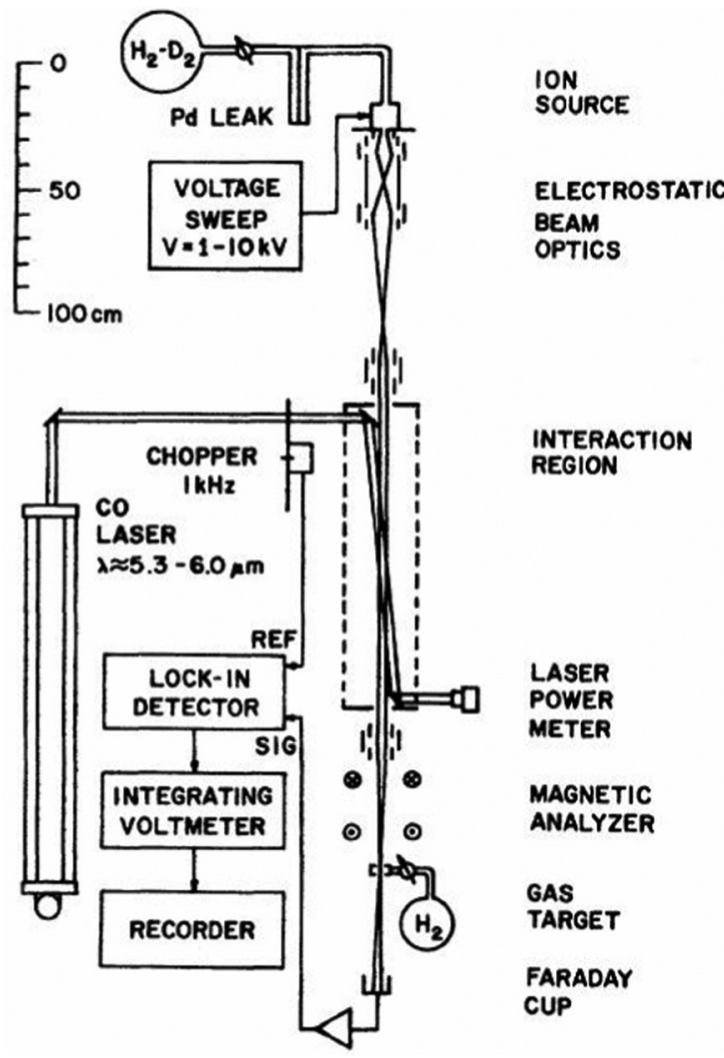

Fig. 2 Doppler-tuned fast-ion-beam laser spectrometer by Wing and coworkers. Reprinted from ref. 58 with permission of APS. 
the detection method. Also, the ions are not counted, but a tiny current measured.

\subsection{Infrared photodissociation - IRPD}

A more advanced method has been developed by Y. T. Lee and collaborators in Berkeley. They used a supersonic expansion source (corona discharge source) to produce cold ionic cores solvated in loosely bound neutral species, such as e.g. $\mathrm{CH}_{5}{ }^{+}\left(\mathrm{H}_{2}\right)_{n}$. These were first mass selected and then stored shortly in a room-temperature ion trap. There, the ions were excited by IR photons into a resonant vibrational state above the lowest dissociation limit, and the subsequent dissociation has been detected by a second mass spectrometer and a Dalydetector. A nonzero lifetime of these resonant states leads to fairly resolvable (ro)vibrational lines and the obtained spectral pattern thus permits to determine the molecular structure. With this 'vibrational predissociation' method, they investigated weakly bound ionic complexes such as $\mathrm{H}^{+}\left(\mathrm{H}_{2} \mathrm{O}\right)_{n}$, $\mathrm{CH}_{5}{ }^{+}\left(\mathrm{H}_{2}\right)_{n}$ or $\mathrm{H}_{3}{ }^{+}\left(\mathrm{H}_{2}\right)_{n}{ }^{25,26,62-64}$ In the so-called messenger (or tagging) variant of this approach, a weakly bound rare gas atom is attached to a stable ion under investigation. ${ }^{32,33,65-68}$ Dissociation with one photon leaves the ion core intact and only leads to the loss of the weakly bound tag. In the limit of large cations and very weakly interacting tags (e.g. He, $D_{0}<$ $1 \mathrm{~kJ} \mathrm{~mol}^{-1}=83.6 \mathrm{~cm}^{-1}$ ), only a minor perturbation is imposed on the structure and spectrum of the core ion, rendering this approach a valuable tool when other vibrational action spectroscopic methods are not available. ${ }^{32,33,35,65,69}$ Instead of using a one-photon process to destroy a tagged ion, a complementary approach is to use infrared multi-photon dissociation (IRMPD) of the naked ion (without tag) in a short intense laser pulse. ${ }^{29,30,70}$

\subsection{Laser induced reactions - LIR}

The action spectroscopic method of Laser Induced Reaction (LIR) has been pioneered by Schlemmer and Gerlich end of the 1990s in Chemnitz. As the name suggests, laser excitation of a trapped molecular ion ensemble enhances the reactivity with a suitable neutral reaction partner, which is added as a gas to the cold ion trap. The spectroscopic signal is then obtained by counting the laser induced product ions as a function of the laser frequency. LIR was first demonstrated with the endothermic $\left(0.18 \mathrm{eV}=1452 \mathrm{~cm}^{-1}\right)$ charge transfer reaction $\mathrm{N}_{2}^{+}+\mathrm{Ar} \rightarrow$ $\mathrm{Ar}^{+}+\mathrm{N}_{2} \cdot{ }^{37}$ The capability to record (a part of) the known Meinl band spectrum $\left(\mathrm{A}^{2} \Pi_{\mathrm{u}} \leftarrow \mathrm{X}^{2} \Sigma_{\mathrm{g}}\right)$ of $\mathrm{N}_{2}{ }^{+}$at low temperature was not the only success of this approach, but, due to the bimolecular collision involved in the detection processes, also statespecific kinetic parameters have been determined. By following the trapped products and reactants in time, and by varying parameters such as the Ar number density or the laser irradiation time, based on a detailed rate equation system, the rotational relaxation rate coefficient in the ground state of $o-\mathrm{N}_{2}^{+}\left(\mathrm{F}_{1}\right.$ component $)$ has been determined, as well as the reaction rate coefficient for vibrationally excited $\mathrm{N}_{2}{ }^{+}$ $\left(v^{\prime \prime}>0\right)+$ Ar. $^{37}$
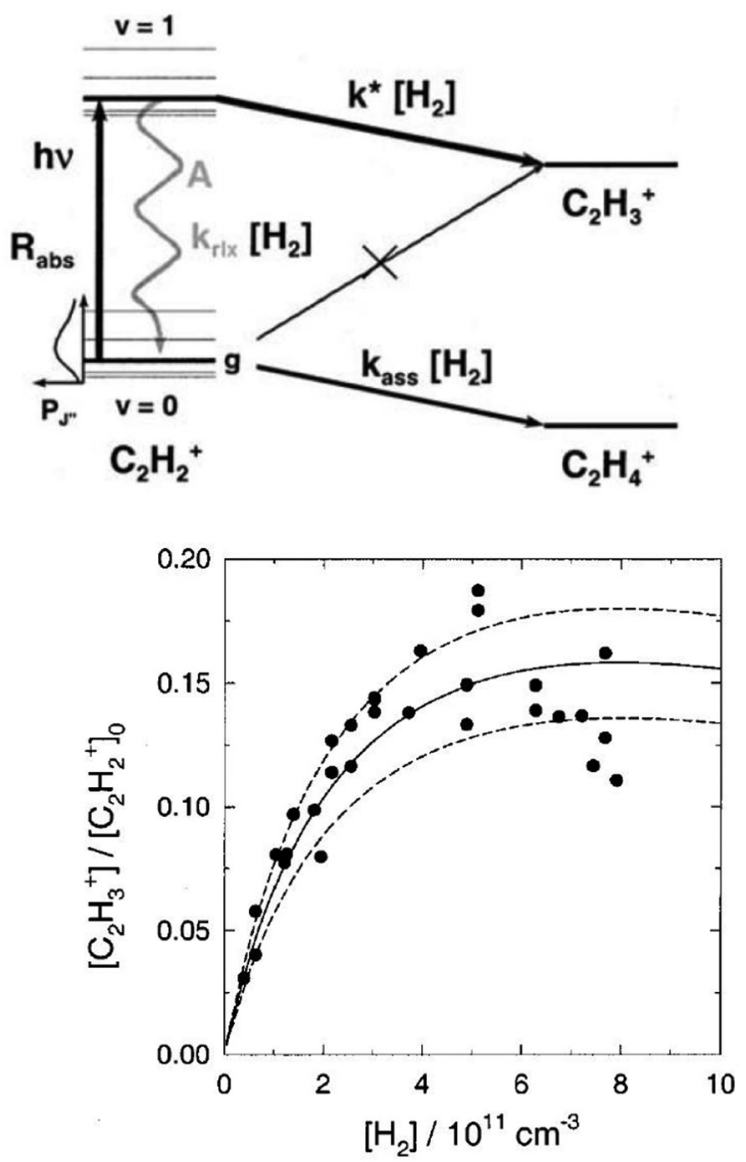

Fig. 3 (top) Level scheme for the LIR-process of $\mathrm{C}_{2} \mathrm{H}_{2}{ }^{+}$showing all radiative and collisional rates. (bottom) LIR-signal for the $P(9)$ transition as a function of the $\mathrm{H}_{2}$ number density, including three different solutions of the rate equation system. ${ }^{38}$ Figures reprinted from ref. 38 with permission of AIP Publishing.

As LIR forms the basis for the rotational action methods described in Sections 4.1 and 4.2, it is explained here in some detail with the example of the hydrogen abstraction reaction ${ }^{38}$

$$
\mathrm{C}_{2} \mathrm{H}_{2}^{+}+\mathrm{H}_{2} \rightarrow \mathrm{C}_{2} \mathrm{H}_{3}^{+}+\mathrm{H} \quad-50 \mathrm{meV}
$$

by which it was possible to record the antisymmetric $\mathrm{C}-\mathrm{H}$ stretching vibration $\nu_{3}$ of $\mathrm{C}_{2} \mathrm{H}_{2}{ }^{+}$(measured previously in the Oka ion factory ${ }^{71}$ ). The basic processes are shown in the top of Fig. 3. The radiative processes are the state-specific laser excitation with the rate $R_{a b s}$ and spontaneous emission with Einstein coefficient $A$. For $\mathrm{C}_{2} \mathrm{H}_{2}{ }^{+}$in the ground state, a collision with $\mathrm{H}_{2}$ does not lead to reaction, while in the excited state it may react with rate $k^{*}\left[\mathrm{H}_{2}\right]$ to $\mathrm{C}_{2} \mathrm{H}_{3}{ }^{+}$or relax vibrationally with rate $k_{r l x}\left[\mathrm{H}_{2}\right]$. As an additional complication in this system, at low temperatures and high $\mathrm{H}_{2}$ number densities, (radiative and ternary) association to $\mathrm{C}_{2} \mathrm{H}_{4}{ }^{+}$occurs. Using this rate equation system for numerical simulations of measured data allows to extract the underlying kinetic parameters. An example of such a measurement, the dependence of the LIR-signal on the $\mathrm{H}_{2}$ number density, is shown in Fig. 3 (bottom). As seen there, the LIR-signal vanishes for low number densities, because the 
$\mathrm{H}_{2}$ collision rate leading to the LIR-signal has to compete with the radiative decay (given by the Einstein coefficient $A$ ). Such measurements and simulations allowed the determination of $k^{*}$ and $k_{r l x}$. Additionally, by comparing the obtained LIRspectrum with a conventional (calculated) spectrum, a dependence of $k^{*}$ on the rotational quantum number was observed. ${ }^{38}$ In later works, these investigations have been extended to the cis-bending vibration $\nu_{5}$ of $\mathrm{C}_{2} \mathrm{H}_{2}{ }^{+} \cdot 39,72$

\subsection{Laser induced inhibition of He attachment - LIICG}

One obvious limitation of the LIR method lies in the need for an endothermic chemical reaction with a neutral reaction partner. This reaction partner should not condense at the low temperatures of the cryogenic trap. Therefore, $\mathrm{H}_{2}$ is often used, allowing for low-temperature experiments down to about $10 \mathrm{~K}$. These requirements restrict the application of LIR to selected cases.

An alternative and generally applicable action technique, Laser Induced Inhibition of Complex Growth (LIICG), emerged with the advent of ion traps cooled to $4 \mathrm{~K}$, and exploits the fact that excitation of a stored cation can inhibit ternary He-attachment in a cryogenic He-bath. This method was pioneered by Maier and Gerlich in Basel, ${ }^{41}$ using rovibronic excitation of $\mathrm{N}_{2}^{+}\left(\mathrm{A}^{2} \Pi_{\mathrm{u}} \leftarrow \mathrm{X}^{2} \Sigma_{\mathrm{g}}\right.$, the same band that was used for the first demonstration of LIR) to hinder the formation of $\mathrm{N}_{2}{ }^{+}$-He at a nominal trap temperature of $5 \mathrm{~K}$. A short time later, the application of this scheme to rovibrational excitation has been demonstrated with the example of $\mathrm{CH}_{5}{ }^{+}$, as shown in Fig. 4, enabling to decipher this enigmatic molecule for the first time. ${ }^{40,42}$ For a typical C-H stretching mode as shown in Fig. 4, the internal energy of the naked cation is increased by about $E / h c \approx 3000 \mathrm{~cm}^{-1}$, which is much higher than the helium binding which is typically less than $1 \mathrm{~kJ} \mathrm{~mol}^{-1}$ $\left(\simeq 83.6 \mathrm{~cm}^{-1}\right) \cdot .^{35,73,74}$ This makes the lifetime of a complex formed by the excited $\mathrm{CH}_{5}{ }^{+}$and He extremely short, so that no collisional stabilization can happen in a ternary collision at the typical He number densities $\left([\mathrm{He}]=10^{15} \mathrm{~cm}^{-3}\right)$. This leads to a decrease, a dip, of the formed $\mathrm{CH}_{5}{ }^{+}-\mathrm{He}$ complexes upon resonant excitation of $\mathrm{CH}_{5}{ }^{+}$, as seen in Fig. 4. As the only

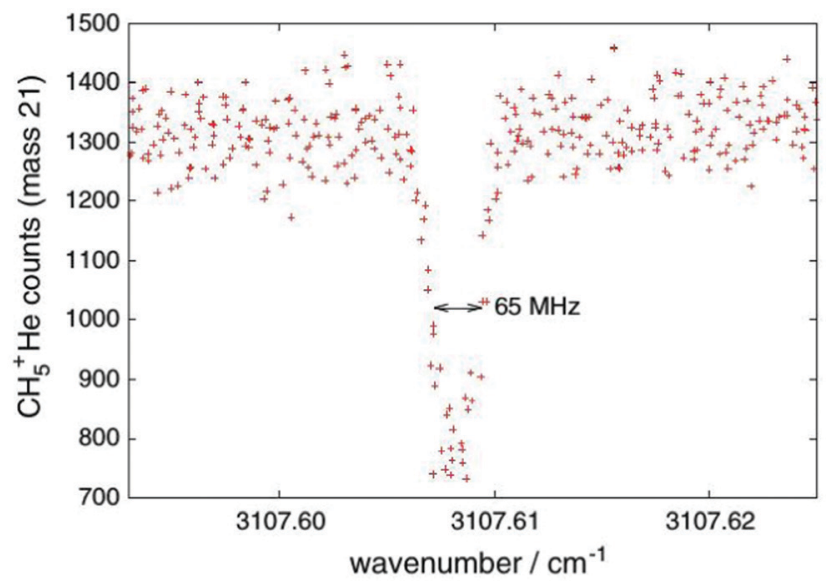

Fig. 4 First vibrational LIICG-signal, demonstrated for the floppy $\mathrm{CH}_{5}{ }^{+}$ ion. ${ }^{42}$ Reprinted with permission of Springer Nature. broadening mechanism at work here is the Doppler effect, this dip has the form of a narrow Gaussian.

The vibrational LIICG method has been successfully applied to many molecular ions. ${ }^{43,44,75-79}$ Typically, this method needs a highresolution cw IR source for effectively shifting the equilibrium between tagged and untagged species (for this equilibrium, see also Section 4.5). Experience shows that pulsed IR lasers do not yield an effective vibrational LIICG signal. For pulsed radiation sources, the induced shift of ion composition in the trap is canceled within a very short time by the competing collisions with He. With the He density as given above and assuming a Langevin collision rate coefficient $k_{L}=10^{-9} \mathrm{~cm}^{3} \mathrm{~s}^{-1}$ and a ternary rate coefficient of $k_{3}=10^{-28} \mathrm{~cm}^{6} \mathrm{~s}^{-1}$, the rate of collisions with He is thus $10^{6} \mathrm{~s}^{-1}$ and the complexes will be formed with a rate $10^{2} \mathrm{~s}^{-1}$. The time scale of complex formation is thus on the order $10 \mathrm{~ms}$ (for this specific example), which is shorter than the time between laser pulses, which is $100 \mathrm{~ms}$ for a typical $10 \mathrm{~Hz}$ laser system. Unless the ion detection is synchronized to happen just after the laser pulse, or using a laser with a higher repetition rate, the effect of the laser excitation will be small.

Very interestingly, rotational excitation, though involving photons carrying very little energy (on the order of only $E / h c \approx 10 \mathrm{~cm}^{-1}$ ), is also able to influence the ternary rate coefficient for He attachment. ${ }^{80}$ The exploitation of this effect for high-resolution rotational spectra of molecular cations is discussed in Section 4.5. A first double resonance experiment using rotational excitation followed by vibrational LIICG is presented in Section 4.6.

\section{Rotational action spectroscopy}

While action spectroscopic techniques for trapped ions and ionic clusters have been available in the vibrational and electronic domain since the 1980 s, first rotational action spectroscopic techniques emerged only one decade ago. This section gives an overview of the different techniques, lists their scope of application (e.g. cation or anion, strongly or weakly bound systems), and compares their experimental parameters.

\subsection{Rotational LIR}

For the direct rotational spectroscopy of a molecular ion using laser induced reaction (LIR) a molecule with large rotational quantum level spacings had to be chosen. Ideal candidates were $\mathrm{H}_{2} \mathrm{D}^{+}$and $\mathrm{D}_{2} \mathrm{H}^{+}$as they fulfill this requirement, have a permanent dipole moment, and their fundamental rotational transitions were well bracketed by previous predictions and experiments. Para $-\mathrm{H}_{2} \mathrm{D}^{+}$, for instance, was known to have its fundamental rotational transition $1_{01} \leftarrow 0_{00}$ at $1.37 \mathrm{THz}^{81,82}$ corresponding to $45.7 \mathrm{~cm}^{-1}$ (or $65.75 \mathrm{~K}$ or $218.813 \mu \mathrm{m})$. In addition, the proton exchange reactions necessary for performing LIR,

$$
\begin{aligned}
\mathrm{H}_{2} \mathrm{D}^{+}+\mathrm{H}_{2} & \rightarrow \mathrm{H}_{3}^{+}+\mathrm{HD} \quad-232 \mathrm{~K} \\
\mathrm{D}_{2} \mathrm{H}^{+}+\mathrm{H}_{2} & \rightarrow \mathrm{H}_{2} \mathrm{D}^{+}+\mathrm{HD} \quad-187 \mathrm{~K} \\
& \rightarrow \mathrm{H}_{3}{ }^{+}+\mathrm{D}_{2}-340 \mathrm{~K}
\end{aligned}
$$




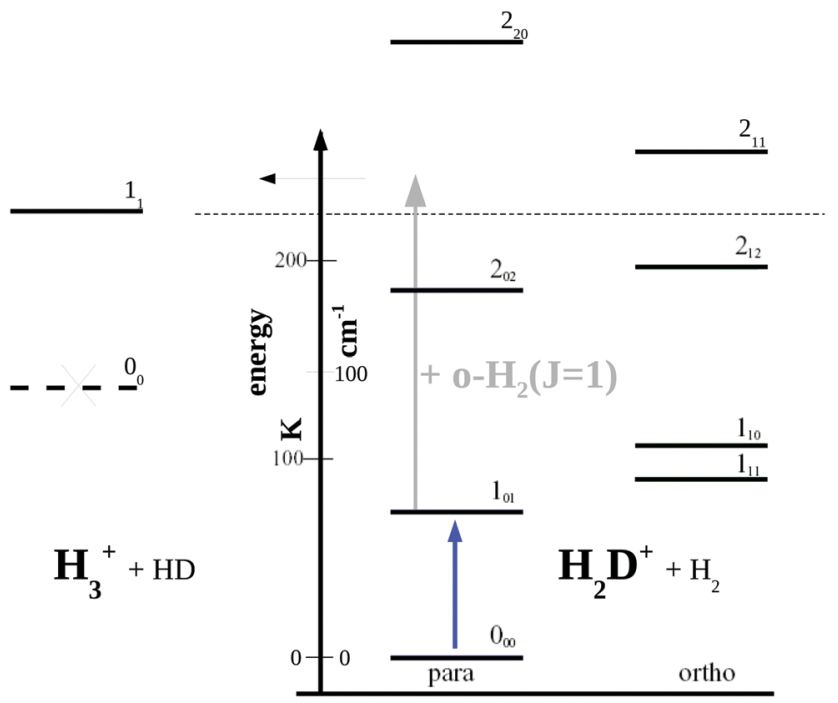

Fig. 5 Rotational levels involved in the low-temperature reaction $\mathrm{H}_{2} \mathrm{D}^{+}+$ $\mathrm{H}_{2} \rightarrow \mathrm{H}_{3}{ }^{+}+\mathrm{HD}$ (reaction (R1)). The lowest level of $\mathrm{H}_{3}{ }^{+}$is $1_{1}$ as the level $\mathrm{O}_{0}$ is forbidden by Pauli exclusion. The given endothermicity (thin dashed line at $232 \mathrm{~K}$ ) is the difference between the lowest rotational levels of the reactant $\mathrm{H}_{2} \mathrm{D}^{+}\left(\mathrm{O}_{00}\right)$ and product $\mathrm{H}_{3}{ }^{+}\left(1_{1}\right)$. The energy scale is given in $\mathrm{K}$ as well as $\mathrm{cm}^{-1}\left(1 \mathrm{~cm}^{-1} \approx 1.4388 \mathrm{~K}\right)$. A similar Figure can be found in ref. 83 .

have energetic barriers which are on the order of the THzexcitation (the given calculated enthalpies are from ref. 82). For the example of reaction (R1), the involved rotational quantum levels, the THz-excitation at $45.7 \mathrm{~cm}^{-1}$, and the endothermicity are summarized in Fig. 5 .

Looking at Fig. 5, one might wonder how the rotational action spectroscopy via LIR works for $\mathrm{H}_{2} \mathrm{D}^{+}$(and $\mathrm{D}_{2} \mathrm{H}^{+}$), as the energy of the THz-photon is only a fraction of the energy barrier. The energy barrier for the reaction $\mathrm{H}_{2} \mathrm{D}^{+}+\mathrm{H}_{2}$ is estimated to be about $232 \mathrm{~K}$ (thin dashed line in the Figure), while the $1_{01} \leftarrow 0_{00}$ THz-photon contributes only $65.75 \mathrm{~K}$ additional energy to the reaction system (blue vertical arrow), leaving a deficit of about $166 \mathrm{~K}$. Also the kinetic energy of the reaction partners is negligible at the low-temperature condition of the ion trap (on the order of $3 / 2 k T \approx 23 \mathrm{~K}$ ). So a very important energy contribution in reaction (R1) stems from $o-\mathrm{H}_{2}$ contained in the hydrogen reaction partner. $o-\mathrm{H}_{2}$ is exclusively in the rotational state $J=1$ at low trap temperature and thus carries an energy content of $170.476 \mathrm{~K}$ (grey arrow in Fig. 5), which finally drives the reaction to the detected product $\mathrm{H}_{3}{ }^{+}$.

First test experiments along these lines were conducted at the FELIX facility (Free Electron Laser for Infrared eXperiments ${ }^{84}$ ), in Neuwegein, the Netherlands (now moved to Nijmegen). This source is capable to produce pulsed THzradiation with its long-wavelength FELIX I undulator (range 67-330 $\mathrm{cm}^{-1}$ ). Previous experiments with FELIX II (range $230-3300 \mathrm{~cm}^{-1}$ ) in 2005 showed successfully ${ }^{83}$ that reaction (R1) can be induced with the excitation of the $\nu_{2}$ and $\nu_{3}$ vibrational bands of $\mathrm{H}_{2} \mathrm{D}^{+}$with origins at 2335.45 and $2335.45 \mathrm{~cm}^{-1},{ }^{85}$ respectively. For the rotational test experiments, conducted in 2006, the same trap setup was used, with an HDPE window for admitting the radiation. The experiments were performed with $n-\mathrm{H}_{2}$ at a nominal temperature of about $15 \mathrm{~K}$. As $\mathrm{H}_{2} \mathrm{D}^{+}$has its dipole moment along its $a$-axis, it exhibits a-type transitions with selection rules $\Delta K_{a}=0( \pm 2, \ldots)$ and $\Delta K_{c}= \pm 1( \pm 3, \ldots)$. The transitions $2_{12} \leftarrow 1_{11}$ at $78.829 \mathrm{~cm}^{-1}$ $(=126.856 \mu \mathrm{m}),{ }^{86} 2_{02} \leftarrow 1_{01}$ at $85.951 \mathrm{~cm}^{-1}(=116.345 \mu \mathrm{m}),{ }^{87}$ $2_{11} \leftarrow 1_{10}$ at $103.483 \mathrm{~cm}^{-1}(=96.635 \mu \mathrm{m})^{87}$ and $2_{20} \leftarrow 1_{01}$ at $178.155 \mathrm{~cm}^{-1}(=56.131 \mu \mathrm{m})^{2}$ (see Fig. 5 for quantum levels) were probed, whereas the fundamental transition at $45.7 \mathrm{~cm}^{-1}$ (blue arrow in Fig. 5) was below the range accessible by FELIX I. No conclusive signals could be detected for these transitions. The nondetection was probably caused by the combination of several reasons, the most important ones being the pulsed structure and limited resolution of the FELIX light source, the use of $n-\mathrm{H}_{2}$ in the experiment (leading to a high background count of $\mathrm{H}_{3}{ }^{+}$ions), as well as the non-optimal partitioning of the probed quantum levels at a nominal trap temperature of $15 \mathrm{~K}$.

For these reasons, the rotational spectroscopy of $\mathrm{H}_{2} \mathrm{D}^{+}$(and $\mathrm{D}_{2} \mathrm{H}^{+}$) has been later continued in the Cologne laboratories with the same trap machine, but using a high-resolution multiplier chain as THz-source, and a vacuum-proof optical CVD diamond window. The setup is shown in Fig. $6{ }^{45}$
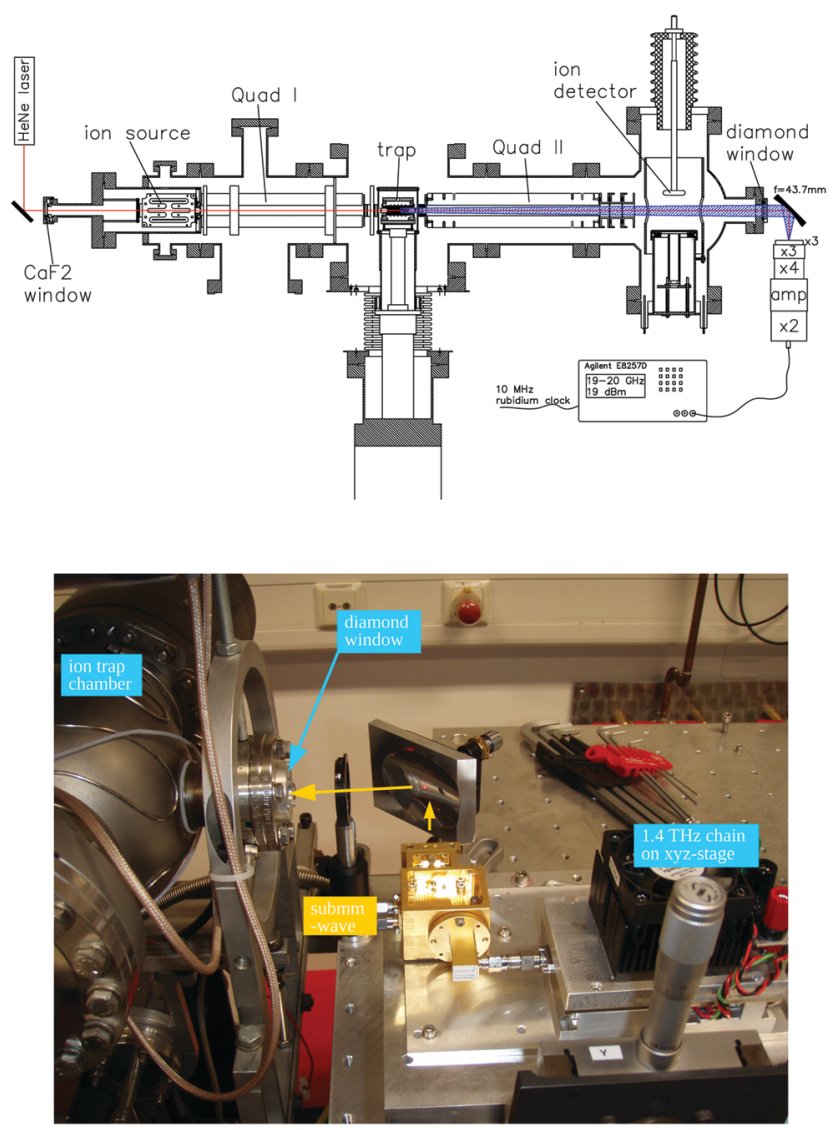

Fig. 6 (Upper scheme) Experimental setup for rotational action spectroscopy of $\mathrm{H}_{2} \mathrm{D}^{+}$and $\mathrm{D}_{2} \mathrm{H}^{+}$, consisting of the ion trapping machine and the $\mathrm{THz}$ source. (bottom photo) The unit consisting of $\mathrm{THz}$ source and ellipsoidal mirror is placed on a common support (simple bread board), which has been moved between the trapping machine and a test setup on a nearby optical bench for adjustment and power calibration. The scheme is reprinted from ref. 45 with permission of APS. 


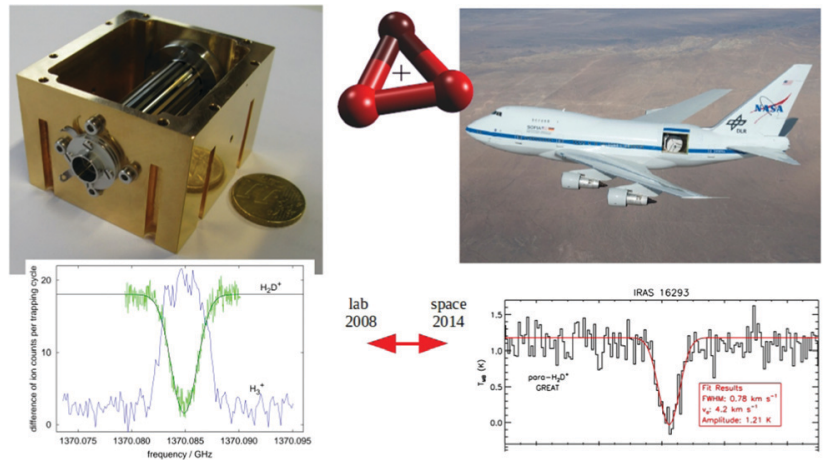

Fig. 7 (Left) Action spectrum of the $\mathrm{H}_{2} \mathrm{D}^{+}$rotational transition $1_{01} \leftarrow 0_{00}$ (blue arrow in Fig. 5), observed as a decrease of the $\mathrm{H}_{2} \mathrm{D}^{+}$parent species and a simultaneous increase of the $\mathrm{H}_{3}{ }^{+}$product ion counts when the excitation frequency is in resonance. ${ }^{45}$ (right) Detection of this transition towards IRAS 16293 with the airborne far-infrared observatory SOFIA. ${ }^{88}$

The multiplier chain, consisting of a doubler, an intermediate amplifier, a quadrupler, and two triplers, giving a total frequency multiplication factor of 72 , operated in the range 1.25 to $1.53 \mathrm{THz}$ with a frequency-dependent output power varying between 1 and $3 \mu \mathrm{W}$. It was estimated that a maximum of about $0.5 \mu \mathrm{W}$ is reaching the trap which is in $0.6 \mathrm{~m}$ distance to the diamond vacuum window. Because of this distance a careful and tedious adjustment of the THz-setup on an optical test bench was mandatory. The experience with these first rotational measurements lead finally to the novel ion trap machine designs developed in Cologne. ${ }^{42,52}$ Also, a hydrogen sample highly enriched in $p-\mathrm{H}_{2}$, produced in an external catalytic $p-\mathrm{H}_{2}-$ generator, has been used in the experiment. With this hydrogen sample, the lower rotational energy content of $\mathrm{H}_{2}\left(J=0\right.$ for $p-\mathrm{H}_{2}$ at low temperature) leads to fewer $\mathrm{H}_{3}{ }^{+}$background counts, and the $p-\mathrm{H}_{2} \mathrm{D}^{+}$under investigation is enriched by collisions with $p-\mathrm{H}_{2}$ in the ion trap. With these prerequisites, the fundamental transitions of $p-\mathrm{H}_{2} \mathrm{D}^{+}$and $o-\mathrm{D}_{2} \mathrm{H}^{+}$could be detected unambiguously for the first time, see Fig. 7 (left). A fit with Doppler profiles yielded line center frequencies of $1370084.880(20) \mathrm{MHz}$ for the $1_{01} \leftarrow 0_{00} p-\mathrm{H}_{2} \mathrm{D}^{+}$transition and $1476605.708(15) \mathrm{MHz}$ for the $1_{11} \leftarrow 0_{00} o-\mathrm{D}_{2} \mathrm{H}^{+}$transition, and thus a relative precision of $\Delta \nu / \nu=10^{-8}$. With these solid laboratory data, these transitions have also been detected in space a few years later, ${ }^{88,89}$ using the airborne far-infrared observatory SOFIA (Fig. 7 right). Of particular interest in these detections was the first determination of the ortho-to-para $(o / p)$ ratio of $\mathrm{H}_{2} \mathrm{D}^{+}$ and $\mathrm{D}_{2} \mathrm{H}^{+}$towards the observed cold protostellar core. Due to the dependence of these $o / p$-ratios on the $o / p$-ratio of the abundant $\mathrm{H}_{2}$ and its thermal history, these ratios are an important indicator of the age of such a region undergoing star-formation.

\subsection{Rotational-vibrational double resonance via LIR}

The THz-measurements of $p-\mathrm{H}_{2} \mathrm{D}^{+}$and $o-\mathrm{D}_{2} \mathrm{H}^{+}$described in the preceding section are the first pure rotational lines detected by action spectroscopy. Despite this success it has again to be emphasized that only accidentally advantageous molecular parameters made the experiment feasible: (i) low-temperature endothermic reactions schemes (R1-R3) exist for $\mathrm{H}_{2} \mathrm{D}^{+}$and $\mathrm{D}_{2} \mathrm{H}^{+}$and (ii) the energy barriers which have to be surmounted in these reactions are relatively low due to the proton exchange character. Typically, other reactions have transition states with much higher barriers. (iii) $\mathrm{H}_{2} \mathrm{D}^{+}$and $\mathrm{D}_{2} \mathrm{H}^{+}$are molecules with large rotational spacings lying in the THz-range. Therefore the excitation of these transitions by THz-photons increases the energy available for the reaction considerably, enabling action spectroscopy to enhance the named reactions. (iv) The THzbeam with its short wavelength can be easily guided from the submm-wave source through the small-diameter diamond window to the $0.6 \mathrm{~m}$ distant ion trap (see Fig. 6 top). Guiding a mm-wave beam $(\sim 100 \mathrm{GHz})$ through the same setup will be considerably more difficult.

For the above reasons, a generalization of this rotational action spectroscopic method to other molecular systems is not possible. For instance, a similar scheme for the heavier ion $\mathrm{CH}_{2} \mathrm{D}^{+}$involves a reaction endothermicity of $370 \mathrm{~K},{ }^{90,91}$ but the fundamental rotational transition $1_{01} \leftarrow 0_{00}$ of $p-\mathrm{CH}_{2} \mathrm{D}^{+}$has a small energy difference, corresponding to $278691.7708(9) \mathrm{MHz},{ }^{92}$ or $13.375 \mathrm{~K}$ only. Alternatively, transitions of $\mathrm{CH}_{2} \mathrm{D}^{+}$in the $\mathrm{THz}$ domain correspond to higher-lying rotational levels (e.g. $5_{24} \leftarrow 4_{23}$ at $1.341 \mathrm{THz}$ ) which are hard to populate at reasonably low temperatures. Early single photon experiments with $\mathrm{CH}_{2} \mathrm{D}^{+}$along these lines in 2009 thus failed. Therefore, other action schemes had to be devised. Doubleresonance experiments including ro-vibrational or even ro-vibronic transitions have been suggested as a solution to this problem already in the original LIR publication. ${ }^{45}$ Such double-resonance schemes have been known to be a powerful tool in the form of IRUV double resonance to obtain vibrational spectra of cold neutral ${ }^{93}$ as well as ionic molecules ${ }^{94}$ via the vibronic excitation as the detection tool.

The rotational-vibrational double resonance method is explained in the upper parts of Fig. 8 for the familiar example of $\mathrm{H}_{2} \mathrm{D}^{+}$. In these schemes, it is assumed that the temperature is lower than the typical energy difference between the rotational levels, $k T<\Delta E$, so that the higher levels are much less populated than the lower levels (doing the simplifying assumption of thermal equilibrium, e.g., at $20 \mathrm{~K}$ the $0_{00}$ level contains $77.6 \%$ of all stored $\mathrm{H}_{2} \mathrm{D}^{+}$ions and the $1_{01}$ level only $8.9 \%$ ). Furthermore, IR and (sub)mm-wave radiation is applied simultaneously to the ions. The frequency of the IR photon (red) is kept fixed on a rovibrational transition starting from a rotational level of the vibrational ground state. In combination with $\mathrm{H}_{2}$-collisions, this results in a detectable and constant LIR signal. The sub(mm)-wave photon (blue) then excites a rotational transition starting or ending on the rotational quantum state probed by the IR laser, as depicted on the left and right upper schemes of Fig. 8, respectively. These two combinations thus decrease or increase the LIR signal. Rotational lines can therefore be recorded by scanning the frequency of the (sub)mm-wave source as shown by the examples in the lower parts of Fig. 8.

For the first such rotational-vibrational double resonance experiment ${ }^{95}$ the same transition $1_{01} \leftarrow 0_{00}$ of $\mathrm{H}_{2} \mathrm{D}^{+}$has been 

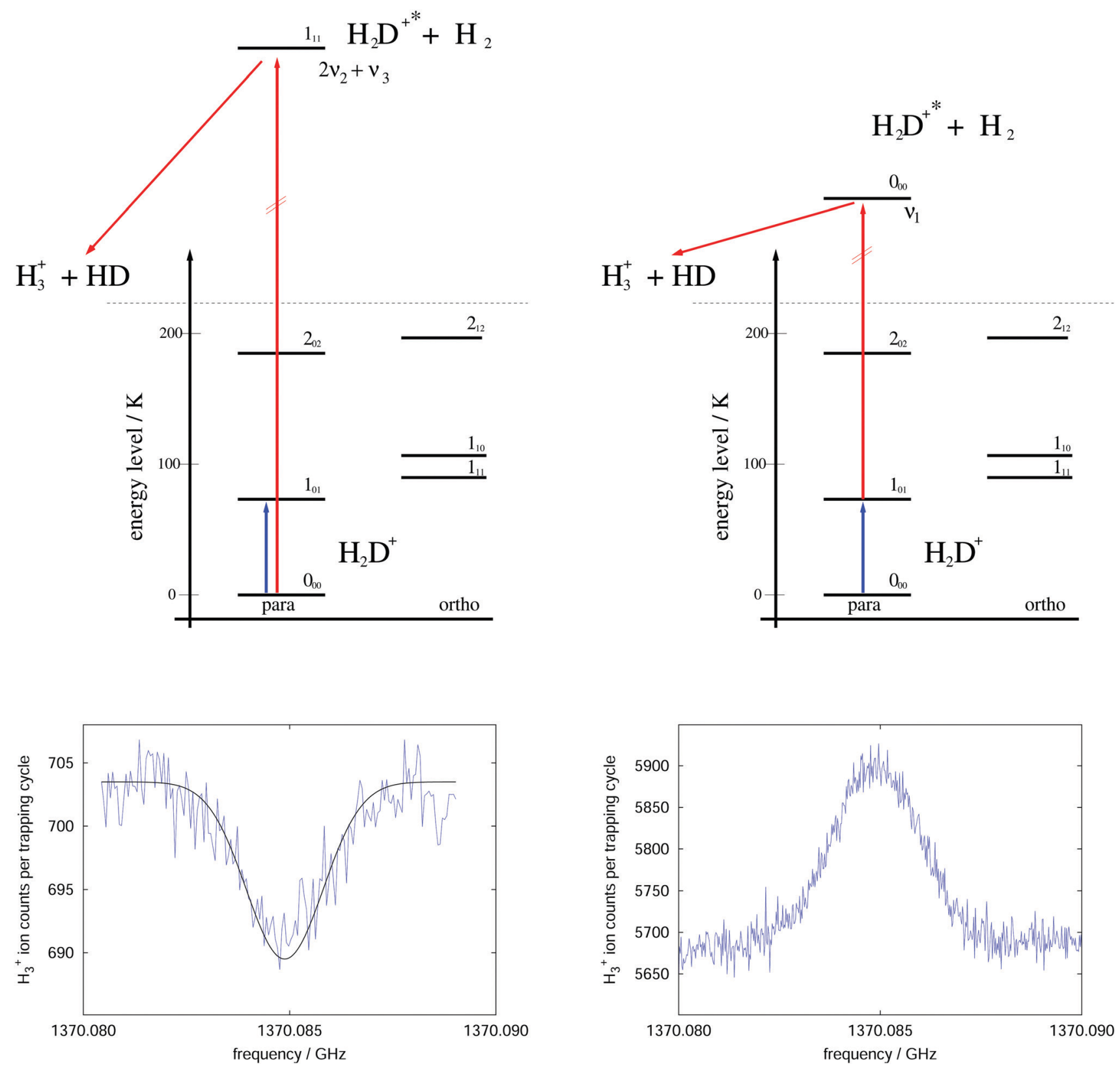

Fig. 8 Rotational-rovibrational double resonance schemes (upper part) and corresponding measurements (lower part), demonstrated on the transition $1_{01} \leftarrow 0_{00}$ (blue arrow) of $\mathrm{H}_{2} \mathrm{D}^{+}$. The left side shows the first demonstration of such a scheme in 2010 (published as Fig. 8 in ref. 95), measured at a nominal trap temperature of $15 \mathrm{~K}$. The rotational excitation creates a dip in the LIR signal which has been generated by excitation (red arrow) into the overtone band $(0,2,1)$. The right side uses the scheme that enhances the LIR signal. The shown curve is the average of four measurements performed at $8 \mathrm{~K}$. The LIR signal has been generated by excitation (red arrow) into the fundamental band $(1,0,0){ }^{96}$

chosen again as a test case, because all involved transitions had been well characterized previously. In particular, the $1_{11} \leftarrow 0_{00}$ IR transition into the overtone band $\left(v_{1}, v_{2}, v_{3}\right)=(0,2,1)$ at $6466.532 \mathrm{~cm}^{-197}$ has been chosen as the rovibrational excitation, driven by a commercially available diode laser (Agilent 8164A diode laser controller with laser module 81642A). The setup was identical with the one shown in Fig. 6, with the diode laser replacing the shown HeNe-laser. The level scheme and the original measurement of this first demonstration are shown in the left part of Fig. 8. A fit to this measured dip with a Gaussian profile yielded a center frequency of $1370084.861(40) \mathrm{MHz}$ and thus a value in very good agreement with the original result shown in Fig. 7. Such control measurements are useful to check that the low-temperature ion trap approach is free of frequency shifts induced by ion motion, as sometimes observed in discharge tube experiments. On the right side of Fig. 8, in contrast, the very same rotational transition has been measured, but using the proposed enhancement scheme. The LIR signal has been detected with a cw IR OPO (Aculight Argos Model 2400) exciting the $0_{00} \leftarrow 1_{01}$ transition 
into the fundamental vibrational band $\left(v_{1}, v_{2}, v_{3}\right)=(1,0,0)$ at $2946.8011 \mathrm{~cm}^{-1} .^{75,97,98}$ The measurement has been done in a new-generation ion trap machine with bent structure. ${ }^{42}$ Six of such measurements at a temperature of $8 \mathrm{~K}$ could improve the uncertainty of this astronomically important transition by a factor of two, leading to the value $1370084.899(12) \mathrm{MHz}{ }^{96}$

A prerequisite for the application of such a double resonance scheme is a thorough knowledge of the ro-vibrational (or even ro-vibronic) spectroscopy of the molecule under investigation. It has to be pointed out that in contrast to the IR-UV double resonance schemes ${ }^{93,94}$ mentioned above, a continuous scanning of the spectrum of interest (i.e. the rotational spectrum in our case) is not feasible, but for every single rotational line a new pair of rotational and rovibrational transitions has to be adjusted in the experiment. As a reward for this additional complication one obtains an inherent verification of the assignments of all pairs of ro-vibrational and rotational transitions. This check may be of advantage for exotic molecules for which the assignment of rotational quantum states needs confirmation (see the example of $\mathrm{CH}_{3}{ }^{+}-\mathrm{He}$ in Section 4.3). An additional advantage of the double-resonance method is that all low-lying transitions can be accessed with good signal-to-noise ratio (which, in favourable cases can reach values of $\sim 10$ for a single measurement), so that a complete network of transitions is obtained, which allows for the accurate determination of all molecular parameters, and thus accurate predictions of unmeasured higher-lying transitions in the THz regime. This has been extensively exploited for deuterated cations of astrophysical interest (see examples below). In principle, both schemes presented in Fig. 8, with the two transitions in competing order (left scheme) or with the two transitions in successive order (right scheme), can be used for double resonance spectroscopy. For measuring the fundamental transitions, one cools the trap to the lowest possible temperature (typically $8 \mathrm{~K}$ for reactions involving $\mathrm{H}_{2}$ ), leading to a high population in the $0_{00}$ state and lower population in the $1_{01}$ level. Both schemes work in this regime with good signal-to-noise ratio, and the resulting narrow Doppler widths allow to determine the line centers with a precision approaching $\Delta \nu / \nu=10^{-9}$. For higher-lying transitions, one has to heat up the trap slightly $(20$ to $40 \mathrm{~K})$ to obtain sufficient population of the probed levels. In these cases the left scheme may be preferable, as the rovibrational transition probes a lower rotational level (which has higher population) and thus a more stable LIR signal may be established.

The rotational-vibrational double-resonance scheme has been applied to many fundamental molecular ions with a permanent dipole moment, for which a suitable LIR reaction scheme is available. As mentioned above, the very high sensitivity of this scheme typically allows to detect all rotational transitions, also weak ones, covered by the (sub)mm-wave sources. For instance, the fundamental transition $J=1 \leftarrow 0$ of the anion $\mathrm{OH}^{-}$has been measured via the endothermic reaction $\mathrm{OH}^{-}+\mathrm{H}_{2} \rightarrow \mathrm{H}^{-}+\mathrm{H}_{2} \mathrm{O}$, yielding a frequency value of 1123101.0410(14) $\mathrm{MHz},{ }^{99}$ and improving the former value measured by Matsushima an coworkers ${ }^{100}$ more than hundred times. Also, deuterated species have been extensively investigated via their endothermic proton exchange reactions. These include the species $\mathrm{H}_{2} \mathrm{D}^{+}$and $\mathrm{D}_{2} \mathrm{H}^{+},{ }^{95,96} \mathrm{CH}_{2} \mathrm{D}^{+}$and $\mathrm{CD}_{2} \mathrm{H}^{+},{ }^{76,92}$ and $\mathrm{HCCD}^{+}$(whose analysis is ongoing). These ions are all known or suspected to promote the deuteration of other molecules in cold interstellar clouds, and the double resonance measurements help in their search. The highly accurate value for the $1_{11} \leftarrow 0_{00}$ transition of $o-\mathrm{D}_{2} \mathrm{H}^{+}$at 1476605.7125(47) $\mathrm{MHz},{ }^{96}$ for instance, supported the detection of $o-\mathrm{D}_{2} \mathrm{H}^{+}$in the protostellar binary IRAS 16293-2422 in Ophiuchus with the GREAT receiver onboard SOFIA. ${ }^{89}$ In a similar way, a complete network of 21 rotational transitions for $\mathrm{CH}_{2} \mathrm{D}^{+}$up to $1.1 \mathrm{THz}$ measured by Töpfer et al. ${ }^{92}$ enables the radio-astronomical search of this ion in interstellar space. Its detection seems currently very difficult because of the low predicted abundances, so that future observations require deep integration. Also, a complete set of 25 rotational transitions has been measured for the $\mathrm{CD}_{2} \mathrm{H}^{+}$cation. While this doubly deuterated ion is extremely hard to detect in interstellar space, the interest for its transition frequencies stems from molecular physics, as this ion exhibits perturbations in its vibrational bands. ${ }^{71,76,101}$ Therefore, a thorough characterization of its unperturbed ground vibrational state is desirable. Fig. 9 shows a summary of rotational quantum levels of $\mathrm{CD}_{2} \mathrm{H}^{+}$and the 25 rotational transitions measured by Jusko et $a .^{76}$ (blue arrows). The application of the double-resonance technique allowed for a complete coverage of all transitions below $1.1 \mathrm{THz}$, even weak ones with $\Delta K_{c}=3$ of this asymmetric rotor. With this complete coverage, by forming sums and differences of the measured rotational lines, 43 combination differences within the vibrational ground state could be generated. These combination differences can be used to check the internal consistency of the rotational as well as IR data. For the rotational data, 12 ground state combination differences can be formed in two different

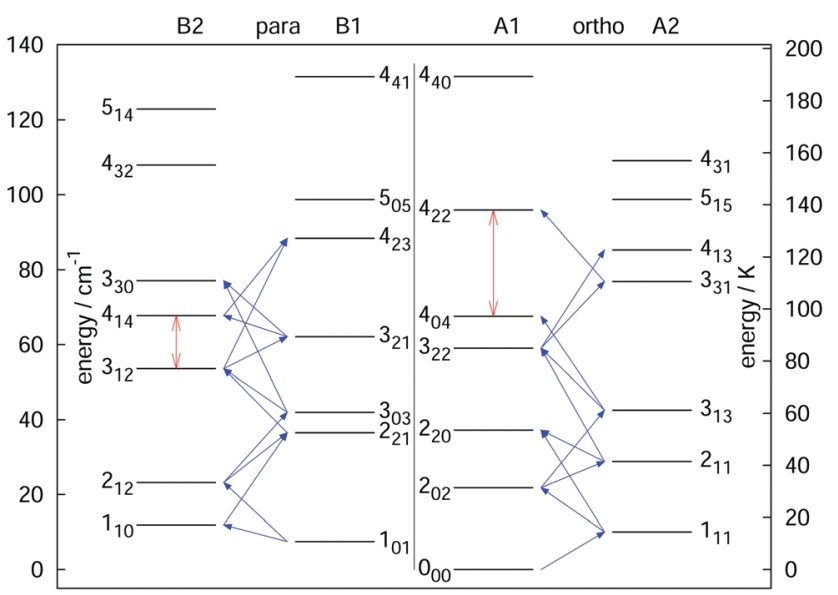

Fig. 9 Illustration of the rotational levels of $\mathrm{CD}_{2} \mathrm{H}^{+}$and the 25 rotational transitions (blue arrows) measured with the double resonance scheme in high resolution. ${ }^{76}$ The red vertical arrows are two examples for combination differences formed by sums or differences of the rotational frequencies (e.g. the $4_{14} \leftrightarrow 3_{12}$ combination difference can be formed by one sum and one difference of the transitions). Such highly accurate combination differences can help to validate IR data of that molecule. ${ }^{76}$ Reprinted with permission of Elsevier. 
ways, and the values of these corroborate the tight accuracies. Also, the 43 combination differences obtained by the rotational measurements can be compared to the ground state combination differences obtained by the highly accurate frequency comb measured IR data of that work. ${ }^{76}$ For example, the difference of the levels $4_{14}$ and $3_{12}$ (see vertical red arrow in Fig. 9) could be formed in two different ways by the rotational data (one difference and one sum, see blue diagonal lines in Fig. 9) and in four different ways by IR combination differences. Plenty of such comparisons can be made with help of the data, forming a consistent set of highly accurate transitions for this molecule.

As a final example, the rotational signatures of the linear ion $\mathrm{HCCD}^{+}$are discussed in the following. Similar to the ions mentioned above, the high-resolution IR spectroscopy work of this ion was pioneered by the Oka group. ${ }^{71,102}$ For the double resonance work, first the $\nu_{1}$ symmetric stretching band of $\mathrm{HCCD}^{+}$was revisited using LIR, for which the band center was determined to be at about $3183.4 \mathrm{~cm}^{-1}$. In contrast to the ions mentioned above, $\mathrm{HCCD}^{+}$is an open shell molecule, with a ${ }^{2} \Pi$ ground state (i.e. with a lone electron with spin $S=1 / 2$ and orbital angular momentum along the molecular axis $\Lambda=1$ ), thus exhibiting spin-orbit interaction and $\Lambda$-doubling. The corresponding rotational levels are depicted in Fig. 10, illustrating the two spin-orbit components $F_{1}\left({ }^{2} \Pi_{3 / 2}\right)$ and $F_{2}\left({ }^{2} \Pi_{1 / 2}\right)$. With the double-resonance method, 8 rotational transitions have been measured within $F_{1}$ between 135 and $519 \mathrm{GHz}$, and 6 within $F_{2}$ (see blue arrows in Fig. 10), each consisting of two $\Lambda$-doubling components. These rotational lines exhibit a pronounced hyperfine structure (not depicted in the Figure) due to the interaction with the nuclear spins of the hydrogen and deuterium nuclei (with nuclear spins of $I=1 / 2$ and $I=1$, respectively). Although the analysis of the extended hyperfine structure is not complete yet, it seems clear at this point that

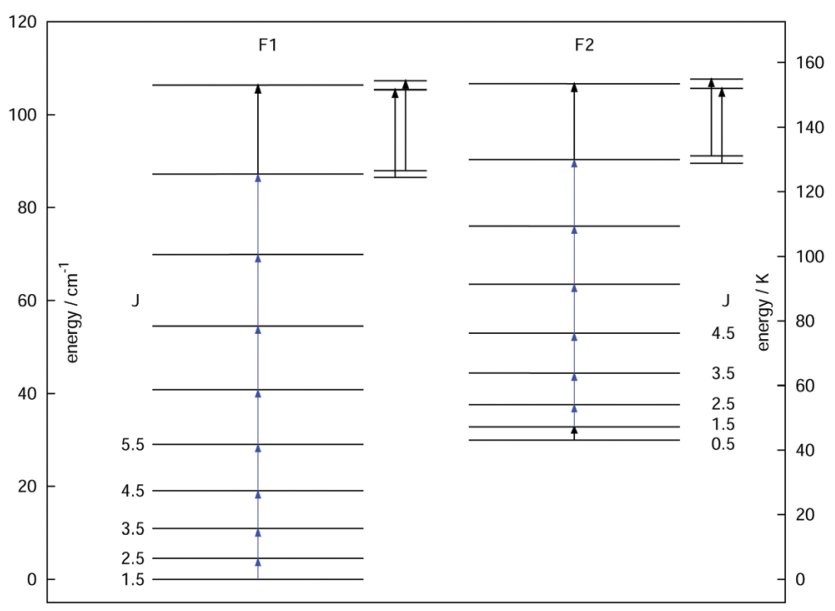

Fig. 10 Illustration of the rotational levels of $\mathrm{HCCD}^{+}$with the two spinorbit components $F_{1}\left({ }^{2} \Pi_{3 / 2}\right)$ and $F_{2}\left({ }^{2} \Pi_{1 / 2}\right)$. The $\Lambda$-doubling is not discernible on this scale, and has been magnified by a factor of 100 for the two highest transitions (see small insets). Fourteen transitions (each with two $\Lambda$-doubling components) have been measured by the doubleresonance method, indicated by the blue arrows. the severe signal dilution for this ion due to the fine and hyperfine structure (and potentially also the Zeeman effect) will make the detection of this ion in space very challenging (apart from its low abundance).

\subsection{Rotational-vibrational double resonance via predissociation}

The preceding section demonstrated that high-resolution rotational spectroscopy can be performed by double resonance via a vibrational action spectroscopy scheme. This means that if a vibrational action spectroscopy scheme with rotational resolution exists for a molecule, the spectroscopy can be readily extended into the rotational domain by such doubleresonance methods. One well-proven vibrational action spectroscopic method is predissociation. In the domain of ionic complexes, it has been applied to fundamental weakly bound systems such as $\mathrm{H}^{+}\left(\mathrm{H}_{2} \mathrm{O}\right)_{n}, \mathrm{CH}_{5}{ }^{+}\left(\mathrm{H}_{2}\right)_{n}$ or $\mathrm{H}_{3}{ }^{+}\left(\mathrm{H}_{2}\right)_{n},{ }^{25,26,62,63,67,103}$ as well as to cation-rare gas complexes ${ }^{104-108}$ at low temperature, and in particular to cation-helium complexes. ${ }^{32,33,35,65,69}$ The first demonstration of the rotational-predissociation double resonance technique has been shown for the $\mathrm{CH}_{3}{ }^{+}-\mathrm{He}$ complex, ${ }^{28}$ based on earlier IR predissociation work by Dopfer and coworkers ${ }^{65}$ in Basel. This test system has been chosen because of its easy production, its presumable simple spectrum (closed shell symmetric top), its large dipole moment $\left(2.9 \mathrm{D}^{101}\right)$, and because its $\nu_{3}$ band (antisymmetric $\mathrm{C}-\mathrm{H}$ stretch) features well-separated rovibrational lines. ${ }^{65}$ The action scheme is illustrated in Fig. 11, and is very similar to the schemes shown in Fig. 8. A tunable narrow-bandwidth (sub)mm-wave beam is overlapped with an IR laser beam. The laser frequency is fixed on one predissociating vibrational transition (one of the red arrows in Fig. 11) to obtain a constant photodissociation signal. Depending on the choice of the vibrational transition combined with the rotational transition (blue arrow), either depletion or enhancement of the signal intensity is obtained, when the (sub)mm-wave source is tuned through the rotational resonance. Please observe that the different signal forms (peak vs. dip) in Fig. 8 and 11 are due the detection of the product ion $\left(\mathrm{H}_{3}{ }^{+}\right.$in Fig. 8) and the parent ion $\left(\mathrm{CH}_{3}{ }^{+}-\mathrm{He}\right.$ in Fig. 11), respectively.

When this rotational method is applied to cation-helium complexes, interesting spectroscopic features may be anticipated due to the large amplitude motion of the loosely bound He atom. Up to date, the symmetric top $\mathrm{CH}_{3}{ }^{+}-\mathrm{He}^{28}$ and the linear $\mathrm{HCO}^{+}-\mathrm{He}^{109}$ have been investigated, for which twentythree and eleven highly resolved rotational lines have been measured, respectively. In both cases, the non-harmonic shallow potential energy surfaces lead to poorly converging spectroscopic fits, so that high orders of centrifugal distortion constants have to be included. In addition, the symmetric top $\mathrm{CH}_{3}{ }^{+}-\mathrm{He}^{28}$ exhibits an unexpected perturbation, which effectively renders the Hamiltonian to be formally that of a slightly asymmetric top. This is detected as splittings for the $K=1$ transitions of the order of $1 \mathrm{MHz}$ (see Fig. 12), which increase with $J$, whereas levels with $K=0$ and $K \geq 2$ have no and negligible splittings, respectively. It has been speculated ${ }^{28}$ that 


$$
\left(\mathrm{v}_{3}, \mathrm{~J}, \mathrm{~K}\right)
$$

$$
(1,1,1)
$$

$(0,2,0)$
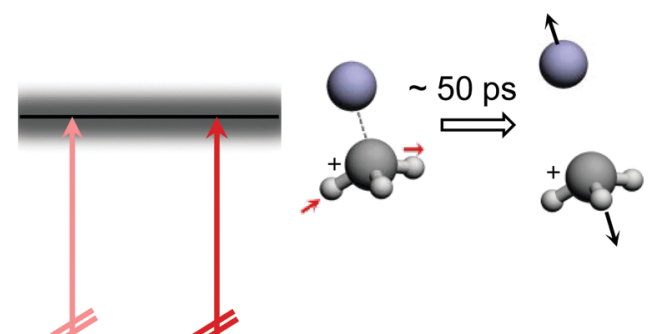

$(0,1,0)$
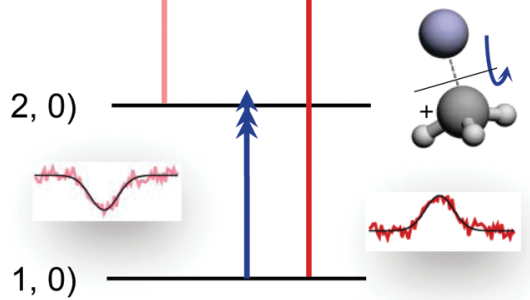

Fig. 11 Rotational-predissociation double resonance scheme illustrated for the example of $\mathrm{CH}_{3}{ }^{+}-\mathrm{He}^{28}$ The fixed IR laser probes either the initial (dark red) or the final (light red) level of the rotational transition (blue), giving rise to different signal forms when the frequency of the rotational excitation is scanned: in the left combination, the rotational transition pumps ions into the level $(0,2,0)$ probed by the IR laser, enhancing the dissociation of $\mathrm{CH}_{3}{ }^{+}-\mathrm{He}$. In the right combination, the rotational transition pumps ions out of the level $(0,1,0)$ probed by the IR laser, thus hindering the dissociation. Reprinted with permission of APS.

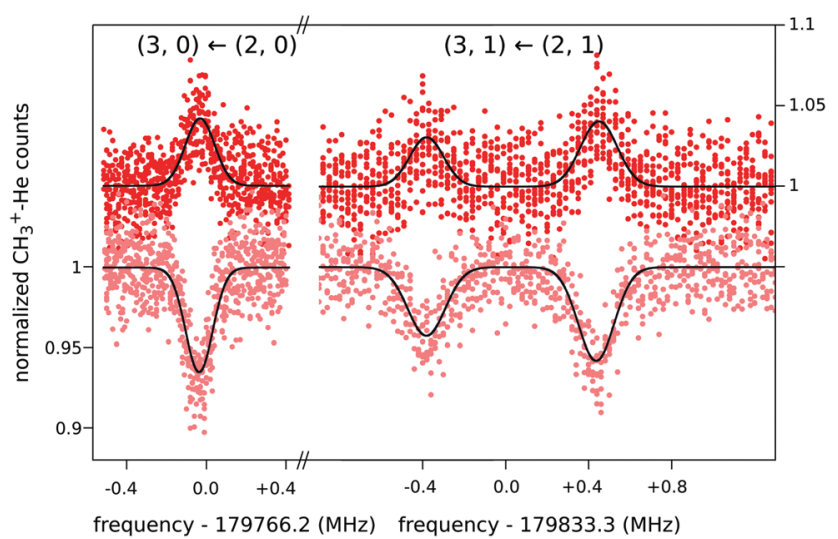

Fig. 12 Four single measurements of rotational ground state transitions of $\mathrm{CH}_{3}{ }^{+}-\mathrm{He}$ at a nominal trap temperature of $4 \mathrm{~K}^{28}$ The color code (light and dark red) corresponds to the two measurement schemes illustrated in Fig. 11. The given quantum numbers $(J, K)$ are those of a symmetric top. The unexpected small splitting for the $K=1$ transition is explained in the text. Reprinted with permission of APS.

this perturbation is caused by the large amplitude motion of the $\mathrm{He}$ atom in the ground vibrational state, with the He atom located on average about $10^{\circ}$ off from the $C_{3}$ symmetry axis,${ }^{65}$ in combination with the internal rotation of the $\mathrm{CH}_{3}$-moiety.

The presented double resonance scheme is widely applicable, as it is based on the fragmentation of a molecule. The only requirements are long-lived resonances in the vibrationally excited state so that individual rovibrational transitions can be picked out by the high-resolution IR laser (typically lifetimes should be longer than $\tau=10$ ps leading to Lorentzian FWHM linewidths of less than $\left.\Delta \tilde{\nu}=1 /(2 \pi \tau) \approx 0.5 \mathrm{~cm}^{-1}\right)$. Advantages of this method are the inherent verification of the assignments of the involved ro-vibrational and rotational transitions (of importance in case of perturbations as described for $\mathrm{CH}_{3}{ }^{+}-\mathrm{He}$ ), as well as the high resolution achieved for Doppler-limited rotational lines measured at cryogenic conditions. Such a high resolution is prevented by lifetime broadening in bare predissociation spectra (this broadening is indicated in Fig. 11 for the predissociating level). On the other hand, the broadened IR lines are beneficial in double resonance experiments, because the requirements for laser stabilization are less strict. Other (action) spectroscopic methods for measuring rotational spectra of weakly bound complexes are less suitable. For instance, measurements of the rotational transitions of $\mathrm{CH}_{3}{ }^{+}-\mathrm{He}$ and $\mathrm{HCO}^{+}-\mathrm{He}$ with the state-selective attachment method described in Section 4.5 failed. $^{28,109}$

The wide applicability of the rotational-predissociation double resonance allows many interesting systems to be investigated in the future. These include more strongly bound complexes such as $\mathrm{CH}_{3}{ }^{+}-\mathrm{Ne}^{106}$ and $\mathrm{CH}_{3}{ }^{+}-\mathrm{Ar},{ }^{105}$ as well as $\mathrm{HCO}^{+}-\mathrm{H}_{2} \cdot{ }^{110}$ Even more advantages can be expected by applying this method to ionic complexes containing nuclei with a quadrupole moment, as e.g. $\mathrm{N}_{2} \mathrm{H}^{+}-\mathrm{He},{ }^{111} \mathrm{NH}_{2}{ }^{+}-\mathrm{He},{ }^{112} \mathrm{~N}_{2}{ }^{+}-$ $\mathrm{He},{ }^{113} \mathrm{DCO}^{+}-\mathrm{He}$ or $\mathrm{ND}_{2}{ }^{+}-\mathrm{He}$. The hyperfine structure can be resolved in these cases, providing additional information on the internal dynamics of the complexes. Further future target systems (possessing a permanent dipole moment) include fundamental complexes such as $\mathrm{CH}^{+}-\mathrm{He},{ }^{44,114} \mathrm{H}_{3}{ }^{+}-\mathrm{He},{ }^{27}$ $\mathrm{H}_{2}{ }^{+}-\mathrm{He},{ }^{115,116}$ or $\mathrm{H}^{+}-\mathrm{He}_{3} \cdot{ }^{74,79,117}$ The latter three complexes are interesting, as they are floppy, consist of only hydrogen and helium atoms, and are thus fundamental few-electron systems. For $\mathrm{H}_{2}{ }^{+}-\mathrm{He}$, although being a fundamental threeelectron system, only difficult-to-assign microwave spectra close to the dissociation limit exist, ${ }^{118,119}$ as well as ab initio data for its rovibrational levels. ${ }^{115,116,120}$ This ion may have played an important role in the early universe, and it still plays an important role in astrochemistry as the intermediate collision complex in the fundamental $\mathrm{He}-\mathrm{H}_{2}{ }^{+}$scattering process. For $\mathrm{H}^{+}-\mathrm{He}_{3}$, a floppy asymmetric rotor of $C_{2 \mathrm{v}}$ symmetry, first experiments to measure its rotational transitions via the presented double resonance scheme in high resolution failed, as the probed proton shuttle motion at $1300 \mathrm{~cm}^{-179,117}$ suffers from substantial lifetime broadening $(\tau \approx 4 \mathrm{ps})$. Future investigations for this molecule will thus target its bending motion at $800 \mathrm{~cm}^{-1}$ in high resolution.

\subsection{Rotational spectroscopy via electron detachment}

Since the first detection of a molecular anion in space, linear $\mathrm{C}_{6} \mathrm{H}^{-}{ }^{12}$ anions have taken a firm place in astrochemical models, and to date six molecular anions have been identified in space based on standard microwave absorption work (see e.g. ref. 14, 17 and 121). For future laboratory detections of anions, 
high-resolution rotational action spectroscopy via double resonance schemes is in principle feasible and has been demonstrated for $\mathrm{OH}^{-99}$ via the reaction given in Section 4.2. But generally it is a fortunate coincidence to find a suitable reaction scheme. Therefore, a more general 2-color action scheme has been developed by Roland Wester and collaborators in Freiburg and Innsbruck, consisting of a sequential resonant (boundbound) absorption of a $\mathrm{THz}$ rotational photon, transferring the anion from a lower rotational state into an upper rotational state, followed by a non-resonant (bound-free) detachment of the excess electron of the anion from the upper rotational state by a tunable laser. In this scheme, the detachment laser is tuned and then fixed so that anions in the upper rotational level are detached, but not the anions in the lower rotational level. Ground for this technique has been laid by former photodetachment spectroscopy work on $\mathrm{OH}^{-},{ }^{122}$ resolving the stepwise opening of additional detachment channels on tuning of the laser, by which the rotational temperature of the trapped and buffer gas cooled anion ensemble could be determined. The neutralization of the anion in the ion trap can thus be taken as the action signal for rotational spectroscopy, which is altered when the $\mathrm{THz}$ frequency is scanned over the rotational resonance. This scheme and the corresponding signals for the transitions $J=1 \leftarrow 0$ and $J=2 \leftarrow 1$ of $\mathrm{OD}^{-}$are presented in Fig. 13. ${ }^{123}$ For the detachment of the excess electron, a cw tunable ring dye laser at $680 \mathrm{~nm}$ has been applied (the detachment threshold for $\mathrm{OH}^{-}$in $J=0$ is $14740.982(7) \mathrm{cm}^{-1122,124}$ ), and for the $\mathrm{THz}$ radiation the difference of two cw NIR diodes has been generated on a photomixer, and the radiation (with a power on the order of several $100 \mathrm{nW}$ ) admitted to the 22-pole ion trap through a Teflon window. The experiment has been performed at a nominal trap temperature of $8.5 \mathrm{~K}$. The Lorentzian signal shape seen in Fig. 13 is given by the spectral shape of the $\mathrm{THz}$ source, with a linewidth of a few $\mathrm{MHz}$, which is in turn given by the linewidth of one of the diode lasers. Lorentzian fits yield center frequencies of $f_{01}=598596.077(188) \mathrm{MHz}$ for the transition $J=1 \leftarrow 0$ and $f_{12}=1196791.573(266) \mathrm{MHz}$ for $J=2 \leftarrow 1$, being in good agreement with previous highresolution discharge tube absorption measurements by Cazzoli and Puzzarini. ${ }^{15,125}$ The obtained uncertainty is on the order of a few $100 \mathrm{kHz}$, and thus a factor of three to six larger than in the previous absorption experiment. However, the demonstration of this action scheme for anion $\mathrm{THz}$ spectroscopy paves the way for the laboratory detection of unexplored molecular anions of astrophysical interest, as a tool complementary to the very successful direct absorption and FT-MW techniques. Due to the non-resonant character of the photodetachment process, a large population difference between the rotational levels is required. The authors estimate that with a future trap temperature of $4 \mathrm{~K}$, rotational transition frequencies as low as $200 \mathrm{GHz}$ become accessible.

While the hydroxyl anion, $\mathrm{OH}^{-}$, is well measured in the laboratory (but not detected in interstellar molecular clouds), the deprotonated form of the interstellar abundant ammonia, the $\mathrm{NH}_{2}{ }^{-}$anion, has been less well characterized. $\mathrm{NH}_{2}{ }^{-}$is an asymmetric top with a singlet electronic ground state, so that its rotational spectrum is not complicated by fine structure. With only rough estimates of its
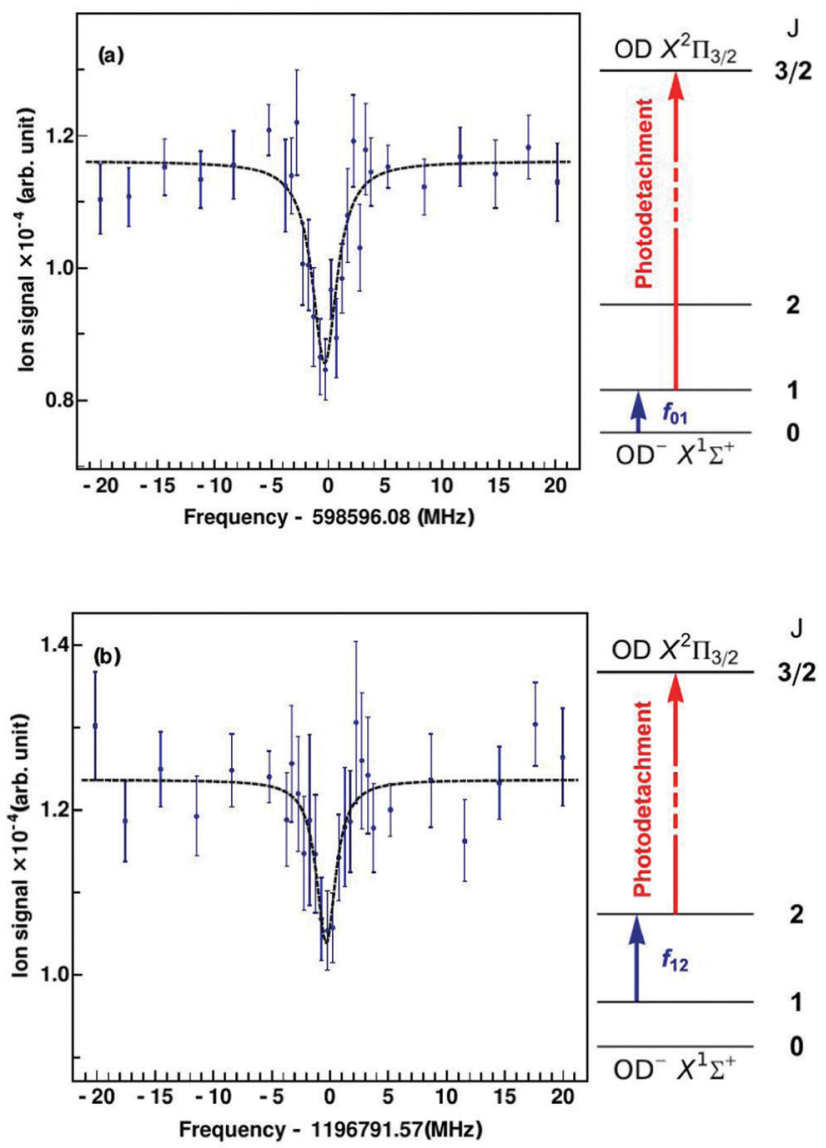

Fig. 13 Rotational action spectroscopy of the $\mathrm{OD}^{-}$anion in a 22-pole ion trap. ${ }^{123}$ On rotational resonance, ions are pumped into the next higher rotational state, shown as blue arrows in the energy level diagrams, $J=1 \leftarrow$ 0 (top, a) and $J=2 \leftarrow 1$ (bottom, b). The increase in population of the upper rotational level is probed using state-selective photodetachment (red arrows), leading to neutralization of the anion in the trap. The data points are obtained from the average of 40 spectral scans. The measured data are fitted using Lorentzian profiles. From the fit, the central frequency is obtained as $f_{01}=598596.077(188) \mathrm{MHz}$ for $\mathrm{J}=1 \leftarrow 0$ and $f_{12}=$ $1196791.573(266) \mathrm{MHz}$ for $\mathrm{J}=2 \leftarrow 1$. Figures reprinted from ref. 123 with permission of APS

rotational transition frequencies at hand, an astronomical absorption feature detected with the HIFI instrument onboard the Herschel satellite towards $\operatorname{SgrB} 2(\mathrm{M})$ at $934 \mathrm{GHz}^{126}$ has been tentatively assigned to this molecule. Recently, the Innsbruck group used the presented rotational-photodetachment spectroscopy scheme to measure the lowest two rotational transitions of deprotonated ammonia, the b-type ortho-transition $1_{10} \leftarrow 1_{01}$ at 447375.1(30) $\mathrm{MHz}$ and the para-transition $1_{11} \leftarrow 0_{00}$ at $933954.2(20) \mathrm{MHz}^{127}$ Due to the low electron affinity of $\mathrm{NH}_{2}\left(6224(1) \mathrm{cm}^{-1128}\right)$, a tunable IR laser near $1600 \mathrm{~nm}$ is sufficient for the detachment of $\mathrm{NH}_{2}{ }^{-}$. Now, with a laboratory frequency value accurate to a few $\mathrm{MHz}$, the authors obtained a discrepancy between the sky and laboratory signal, concluding that the former tentative assignment ${ }^{126}$ is incorrect.

\subsection{Rotational spectroscopy via state-specific He attachment}

As mentioned in Section 4.3, attaching noble gas atoms (traditional 'tags' are argon or neon) or other small molecules to 
ionic species can be exploited for vibrational spectroscopy via predissociation..$^{34,67,129-131}$ This is a very valuable approach in cases where the spectra of the untagged (naked) species are difficult to obtain. Of course, the vibrational transition frequencies of the tagged species are shifted compared to the untagged species, but one obtains good estimations of the vibrational band positions of the naked ion which can then be compared to ab initio calculations. Reducing the influence of the tag can be achieved by attaching helium, as it is much less strongly bound so that much less distortions of the molecular structure and thus smaller shifts in the spectrum can be expected. In the first experiments along these lines, Hetagged clusters have been produced directly in supersonic expansion ion sources, ${ }^{73,132}$ but more recently, modern cryogenic methods allow to produce them in situ in cold ion traps filled with helium gas. ${ }^{33,35,42}$

Such cryogenic ion trap experiments operating close to $4 \mathrm{~K}$ allow an equilibrium of the parent cation and its helium-tagged counterpart to be formed (vide infra). Very interestingly, perturbation of this equilibrium by IR excitation via a cw laser does not only lead to the vanishing of the cation-helium complexes by predissociation, but, by exciting the parent ion, it can also lead to the suppression of the attachment of the loosely bound helium atom, thus making direct spectroscopy of the naked cation feasible. The latter effect, called 'Laser Induced Inhibition of Complex Growth' (LIICG), has been explained in Section 3.5. Cases have been observed, where the predissociation and the LIICG signals of the tagged and untagged species, respectively, are recorded in one and the same scan of the vibrational spectrum. $^{27,44,79}$

The LIICG method as applied to electronic or vibrational spectroscopy can be conveniently explained by an energy argument: the energy absorbed in an electronic or vibrational transition is much higher than the binding energy of the $\mathrm{He}$ atom (which is on the order of $\sim 50 \mathrm{~cm}^{-1}$ ). Therefore, it came as a surprise, when Brünken and Schlemmer pioneered the application of this approach to pure rotational spectroscopy of a cation, where much lower photon energies are involved. The first application of rotational LIICG was demonstrated on the l$\mathrm{C}_{3} \mathrm{H}^{+}$cation, ${ }^{133}$ a linear molecule with a ${ }^{1} \Sigma$ ground state. Four rotational transitions have been measured at a nominal trap temperature of $4 \mathrm{~K}$ in the range 44 to $113 \mathrm{GHz}$, of which one is depicted in Fig. 14. This unambiguous laboratory detection also confirmed the origin of lines which had been previously detected in the Horsehead photodissociation region ${ }^{136}$ and toward Sgr B2(N), ${ }^{137}$ and thus ended discussions about their potential carrier.

Due to the very specific nature of LIICG when applied in the rotational domain, it has been later renamed to 'Rotational state-dependent attachment of $\mathrm{He}$ atoms'. A very detailed account on the kinetics of this process has been given by Brünken et $a .^{80}$ on the example of the fundamental $J=1 \leftarrow$ 0 transition of $\mathrm{CD}^{+}$. This transition as well as the next higher transition $J=2 \leftarrow 1$ are depicted in Fig. 15. In such an experiment, several ten thousand $\mathrm{CD}^{+}$parent ions are stored per trapping cycle in the cold ion trap where they are cooled to

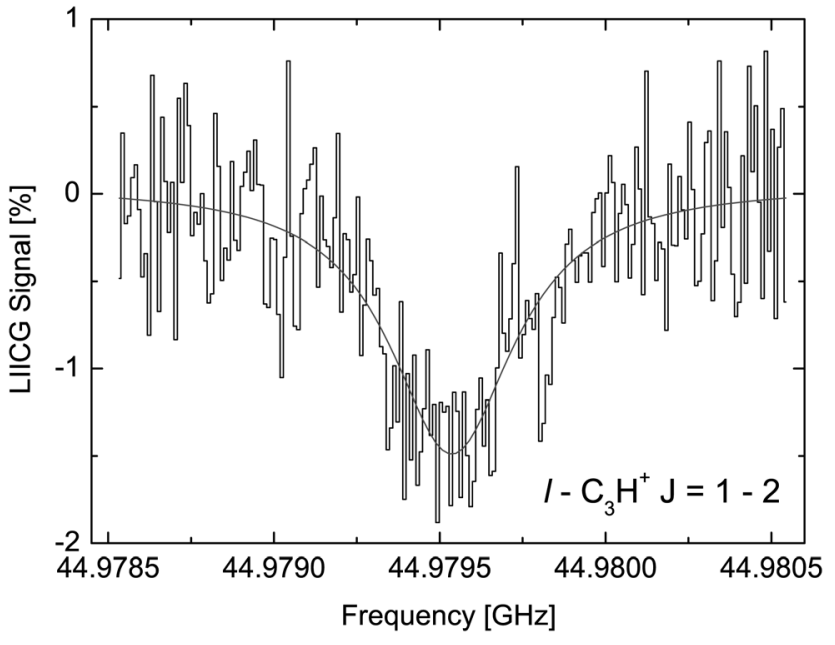

Fig. 14 The rotational transition $\mathrm{J}=2 \leftarrow 1$ of $\mathrm{L}_{3} \mathrm{C}_{3} \mathrm{H}^{+}$measured by storing several ten thousand $\mathrm{C}_{3} \mathrm{H}^{+}$ions in a cold $(4.1 \mathrm{~K})$ and dense $\left(\sim 10^{15} \mathrm{~cm}^{-3}\right)$ helium bath and continuous irradiation with tunable narrow-band $\mathrm{mm}$ wavelength radiation. The rotational transition of the bare ion can be recorded by counting the $\mathrm{C}_{3} \mathrm{H}^{+}-\mathrm{He}$ complex ions as a function of excitation frequency. On resonance, the number of formed complex ions (black histogram) decreases by about 1.5\%. The black line is a fit with a Lorentzian line-shape function to the data. In the original publication, ${ }^{133}$ the lines for $J=3 \leftarrow 2$ and $J=4 \leftarrow 3$ are shown.

the ambient temperature ( $4 \mathrm{~K})$ by collisions with He gas. The helium gas is leaked into the trap in a constant fashion, with the equilibrium He density on the order of $10^{15} \mathrm{~cm}^{-3}$. Owing to these conditions, helium will readily attach to the trapped ions via ternary collision processes. The formed $\mathrm{CD}^{+}-\mathrm{He}$ ion-atom complexes can, on the other hand, be dissociated again by collisions with helium (collision induced dissociation - CID), leading to the equilibrium

$$
\mathrm{CD}^{+}+2 \mathrm{He} \underset{k_{\mathrm{CID}}}{\stackrel{k_{3}}{\leftrightarrow}} \mathrm{CD}^{+}-\mathrm{He}+\mathrm{He} .
$$

To perform rotational spectroscopy of the trapped ions with a cw (sub)mm-wave source, one exploits the fact that the ternary helium attachment rate $k_{3}$ (see equation above) depends on the wavefunction of the state with total angular momentum $J$. Typically, a state with higher $J$ has lower $k_{3}(J)$. For instance, $k_{3}(1)=0.55 \cdot k_{3}(0)$ has been deduced for $\mathrm{CD}^{+}$by comparing measurements to a detailed kinetic model. ${ }^{80}$ The observed effective attachment rate coefficient $k_{3}$ is then a weighted average of state-specific rate coefficients $k_{3}(J)$ with the weights given by the relative rotational state populations $p(J)$ :

$$
k_{3}=\sum_{J} p(J) k_{3}(J) .
$$

As these experiments are operating close to $4 \mathrm{~K}$, only a few occupied quantum levels are involved in this process. Upon excitation of a rotational transition, $J \rightarrow J+1$, the initial Boltzmann-like state population is altered, resulting in a lowering of the effective attachment rate coefficient $k_{3}$. Consequently, the equilibrium given in (1) is shifted to the left, and 

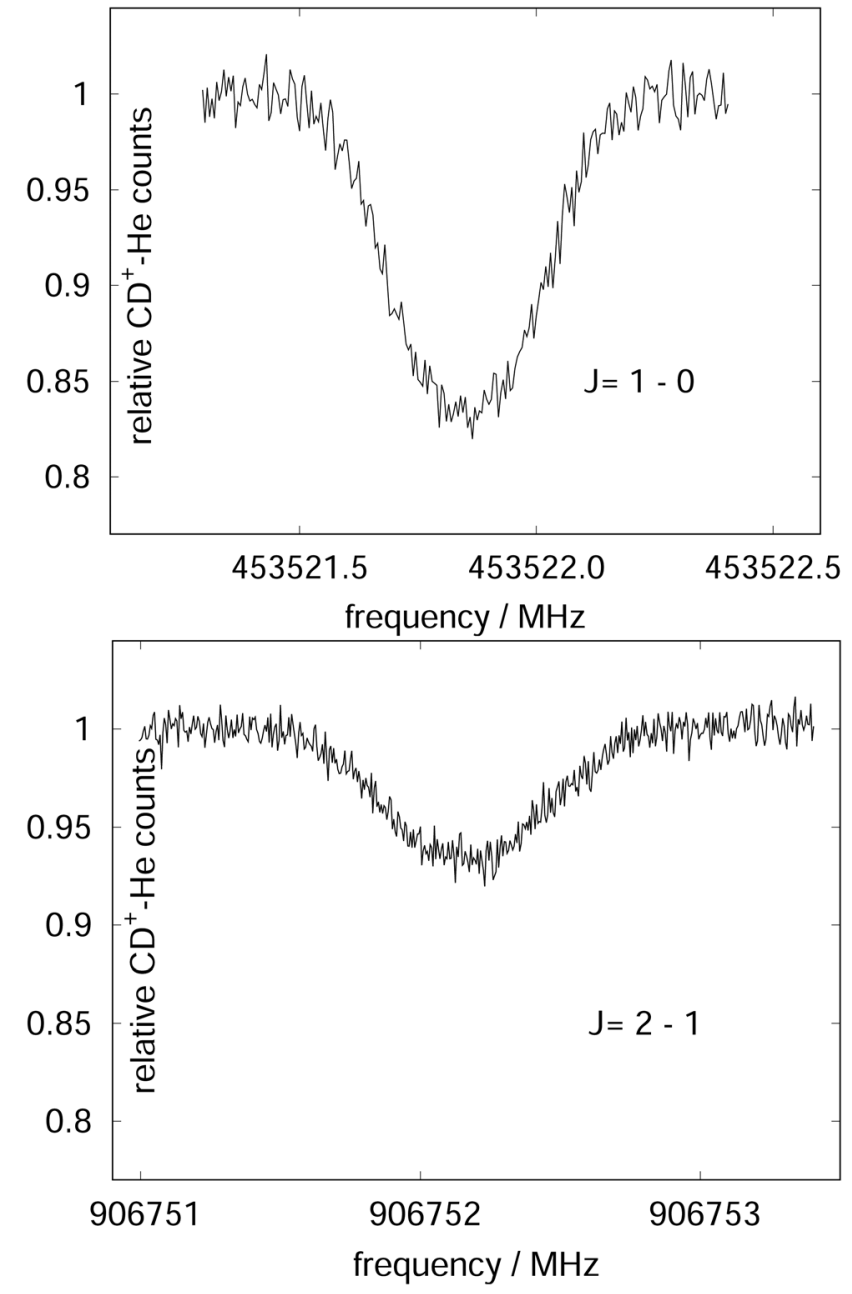

Fig. 15 The rotational transitions $\mathrm{J}=1 \leftarrow 0$ and $\mathrm{J}=2 \leftarrow 1$ of $\mathrm{CD}^{+}$ measured via state-specific He attachment, with depletions of about $17 \%$ and $7 \%$, respectively. Several of such measurements yield the center frequencies 453521.8530(6) and 906752.1649(17) $\mathrm{MHz}$, respectively, improving the uncertainty for the latter transition by nearly two orders of magnitude, ${ }^{134}$ while the accurate value existing for the $J=1 \leftarrow 0$ transition (measured previously in the Cologne laboratories) has been confirmed. ${ }^{80,135}$ Unpublished Figures of the work described in ref. 44.

the number of complex ions $\mathrm{CD}^{+}-\mathrm{He}$ is decreasing on resonance, as depicted in Fig. 15. A complete scheme of these elementary processes is illustrated in Fig. 16, including collisional (inelastic collisions of $\mathrm{CD}^{+}$with $\mathrm{He}$, ternary attachment leading to $\mathrm{CD}^{+}-\mathrm{He}$, and collision induced dissociation of $\mathrm{CD}^{+}-$ $\mathrm{He}$ ) and radiative processes (spontaneous and stimulated radiative rotational transitions), as thoroughly described in ref. 80 .

The presented rotational action spectroscopic scheme needs a high-resolution cw radiation source for effectively shifting the equilibrium given in eqn (1). Pulsed sources are ineffective for this method (similar to LIICG as explained in Section 3.5). Furthermore, the method is confined to trap temperatures close to $4 \mathrm{~K}$, and therefore only a few low-lying rotational levels and transitions can be accessed. But a big advantage is its extreme versatility, as a helium atom can be attached in

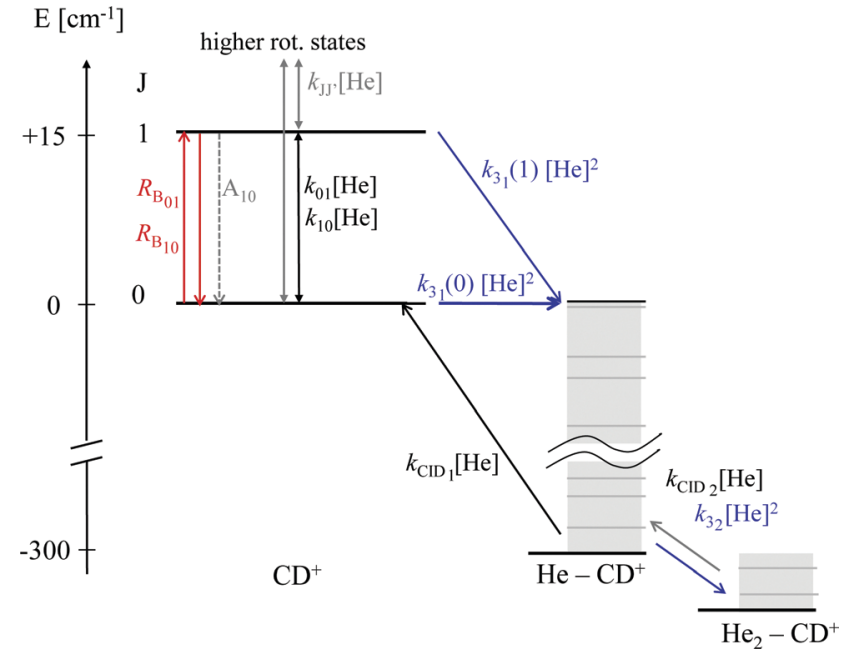

Fig. 16 Scheme of the elementary kinetic processes involved in the ternary attachment of $\mathrm{He}$ to $\mathrm{CD}^{+}$and the simultaneous excitation of the $C D^{+} J=1 \leftarrow 0$ rotational transition. The processes are depicted as arrows and include the rotational state-dependent ternary Helium-cluster formation (blue), the collision induced cluster dissociation (CID, black), the rotational (de-)excitation of the $\mathrm{CD}^{+}$ions by collisions with Helium, the spontaneous emission of the $J=1$ level, and the radiative stimulated (de-)excitation of $\mathrm{CD}^{+}$(red), with the associated rates given by the corresponding symbols. Figure reprinted from ref. 80 with permission of Elsevier.

principle to any cation. More than a dozen molecular cations, for which no other suitable rotational action spectroscopic method could be identified, have been investigated in the laboratory with the state-dependent He attachment method, many for the first time. Exact rotational transition frequencies have been provided for $\mathrm{l}_{-} \mathrm{C}_{3} \mathrm{H}^{+},{ }^{133} \mathrm{CF}^{+},{ }^{138} \mathrm{SiH}^{+},{ }^{139} \mathrm{HCO}^{+},{ }^{109}$ $\mathrm{CD}^{+},{ }^{44,80} \mathrm{CH}^{+}$and ${ }^{13} \mathrm{CH}^{+},{ }^{44} \mathrm{NH}_{3} \mathrm{D}^{+},{ }^{138,140} \mathrm{NH}_{2} \mathrm{D}_{2}{ }^{+}$and $\mathrm{NHD}_{3}{ }^{+140} \mathrm{CN}^{+141} \mathrm{CH}_{2} \mathrm{NH}_{2}{ }^{+},{ }^{77} \mathrm{CH}_{3} \mathrm{NH}_{3}{ }^{+142} \mathrm{NO}^{+143}$ and $\mathrm{CCl}^{+} \cdot{ }^{144}$

To date, attempts to attach a helium atom to an anion at $4 \mathrm{~K}$, e.g. to $\mathrm{OH}^{-},{ }^{99}$ were unsatisfactory, because the involved binding energies are even lower than for complexes consisting of a neutral molecule and He. ${ }^{145}$ For the spectroscopy of anions via this method, traps operating well below $4 \mathrm{~K}$ (with e.g. pulse tube cooling) have to be constructed in the future. But also for cations, there have been failed attempts to record rotational transitions, though He atoms could be attached. As mentioned above, the state-dependent He attachment method relies on the dependence of $k_{3}$ on the quantum state. But one cannot know $a$ priori whether there is such a substantial dependence. Also, the $\mathrm{cw}$ radiation source must be powerful enough to compete with the collisional processes. ${ }^{80}$ There have been cases where the detection via the rotational state-dependent attachment method simply failed because of these reasons. These failed attempts include the rotational spectroscopy of $\mathrm{O}_{2} \mathrm{H}^{+}\left(3 \mathrm{~A}^{\prime \prime}\right),{ }^{43}$ $\mathrm{C}_{3} \mathrm{H}_{2}{ }^{+}\left({ }^{2} \mathrm{~A}_{1}\right),{ }^{146} \mathrm{PH}^{+}\left({ }^{2} \Pi\right)$ and $\mathrm{N}_{2} \mathrm{O}^{+}\left({ }^{2} \Pi\right)^{143}$ as well as the wellpredicted transition $J=1-2$ of $\mathrm{SiH}^{+}\left({ }^{1} \Sigma\right)^{139}$ and the $2_{0}-1_{0}$ and $2_{1}-1_{1}$ transitions of the symmetric rotor $\mathrm{NH}_{3} \mathrm{D}^{+}\left({ }^{1} \mathrm{~A}_{1}\right) \cdot{ }^{138,140}$ Also, very interestingly, for the $N=1-0$ transition of $\mathrm{CO}^{+}\left({ }^{2} \Sigma\right)$, only one fine structure component $\left(1_{3 / 2}-0_{1 / 2}\right)$ could be detected, 
the one in which the total angular momentum quantum number $J=S+N$ changes, but not the other transition $\left(1_{1 / 2}-0_{1 / 2}\right)$ of that doublet. ${ }^{80}$ It is extremely striking that all successful examples of this method have a singlet electronic ground state, while the failed examples above contain many open-shell species. One obvious reason contributing to this finding is that open-shell species exhibit more spectral features and thus suffer from signal dilution. Nevertheless, further investigations on the application of this method to open-shell systems seem mandatory.

\subsection{Rotational-vibrational double resonance via LIICG}

Quite recently, the vibrational version of LIICG (see Section 3.5) has been applied to perform rotational spectroscopy ${ }^{77}$ via double-resonance. This may seem obsolete, as hindering of He attachment can be achieved by a rotational photon alone, as demonstrated in the preceding Section 4.5. But in contrast to electronic or vibrational excitation, the photons involved in molecular rotational transitions have very little energy (on the order of only $E / h c \approx 10 \mathrm{~cm}^{-1}$ ), so the suppression of complex formation upon resonant absorption is rather limited, since the ternary attachment of He is only hindered (or changed) instead of inhibited. To improve the limited depletion signal in a pure rotational excitation, a double resonance scheme which is based on the more pronounced LIICG-signal of the rovibrational excitation has been demonstrated for protonated methanimine, $\mathrm{CH}_{2} \mathrm{NH}_{2}{ }^{+} \cdot{ }^{77}$ The scheme and the results are shown in Fig. 17. For this scheme, the IR excitation frequency of the optic parametric oscillator is held on a rovibrational transition, producing a LIICG depletion signal. This LIICG depletion can then be altered by a rotational transition which pumps additional ions into the rotational state probed by the IR laser. By scanning the mm-wave excitation frequency over the rotational transition frequency while the IR wavenumber is kept fixed on resonance, the rotational line can be recorded. As seen on the right side of Fig. 17, this novel double resonance scheme exhibits a signal up to 2-3 times stronger than the rotationonly method, which enabled the detection of weak pure rotational transitions of the $\mathrm{CH}_{2} \mathrm{NH}_{2}{ }^{+}$molecule. Up to date, this novel method has been applied to $\mathrm{CH}_{2} \mathrm{NH}_{2}{ }^{+}$only, but it seems to be a promising tool in cases where other rotational action schemes failed (e.g. $\mathrm{O}_{2} \mathrm{H}^{+},{ }^{43} \mathrm{C}_{3} \mathrm{H}_{2}{ }^{+146}{ }^{4} \mathrm{HC}_{3} \mathrm{O}^{+}$or the $J=1-2$ transitions of $\mathrm{SiH}^{+},{ }^{139}$ see also preceding section).

\section{Rotational spectroscopy of sympathetically cooled ions}

The examples of the preceding sections showed some quantum state control of the molecular ion ensemble and spectroscopy in high resolution simply by cooling them via helium buffer gas. Even lower kinetic temperatures, and in particular Doppler-free operation, as well as better quantum state control can be obtained by co-trapping molecular ions with lasercooled atomic ions (or even a single molecule with a single atom) in a linear Paul trap (so-called sympathetic cooling). In combination with other techniques, e.g. quantum logic spectroscopy, ${ }^{148}$ very detailed information and highest rotational resolution is obtained, as shown with the two examples of $\mathrm{HD}^{+}$and $\mathrm{CaH}^{+}$in the following. These examples can be considered action spectroscopy. But in contrast to the examples
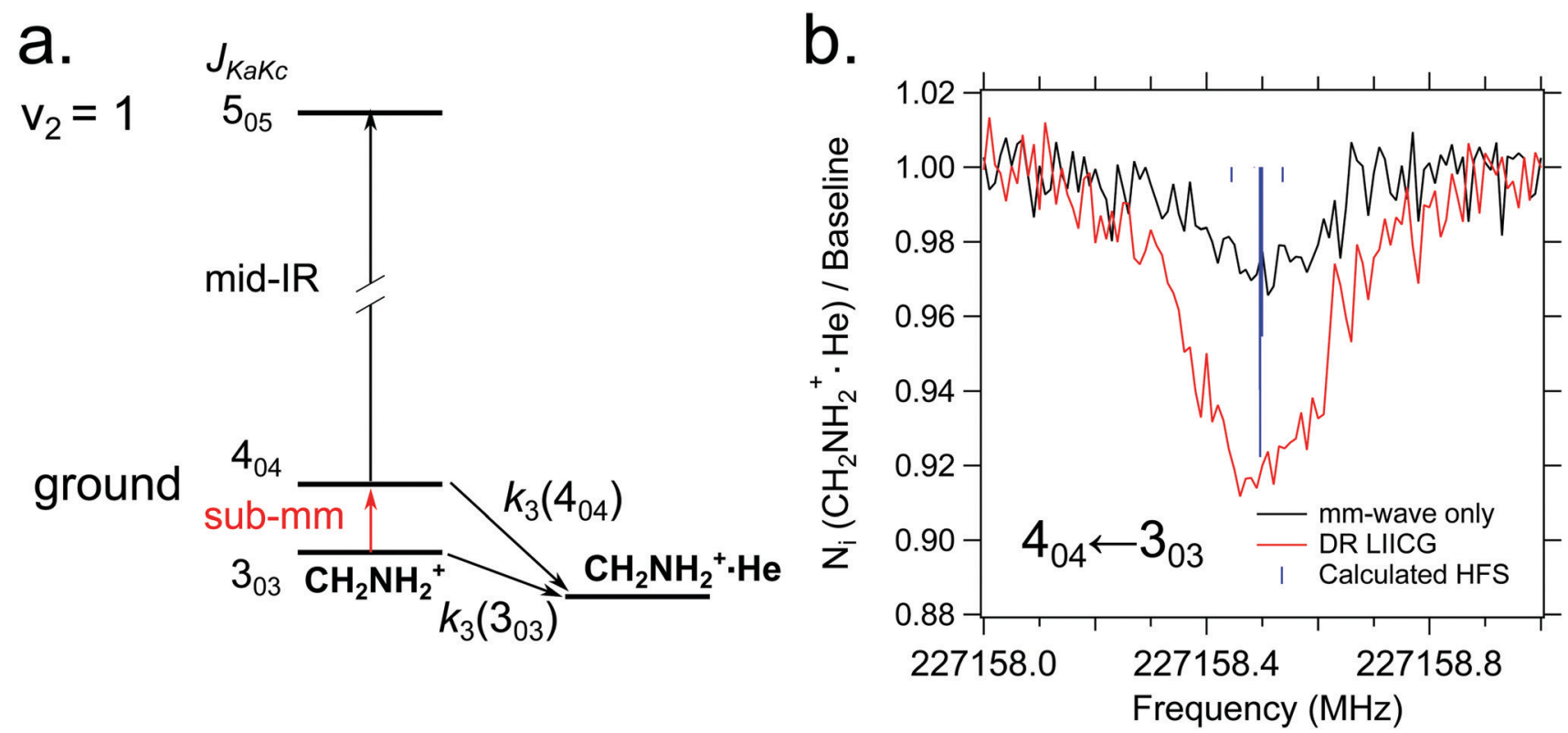

Fig. 17 (a) A simple diagram showing the transitions and tagging mechanism involved in the double resonance scheme for the $\mathrm{CH}_{2} \mathrm{NH}_{2}{ }^{+}$molecule. The ternary association rate with $\mathrm{He}$ is designated $k_{3}$. (b) A comparison between scans of the $4_{04} \leftarrow 3_{03}$ transition taken with standard mm-wave-only scheme (black) and double resonance using LIICG (red), which in this case was stronger by a factor of three. Hyperfine structure from the ${ }^{14} \mathrm{~N}$ nucleus $(I=1)$ is indicated with blue sticks but was not resolved in the spectroscopic measurements. Reproduced from ref. 77 with permission from the PCCP Owner Societies. 
given before, the spectroscopic signal here is not given by counting the ions (as shown in Fig. 1), but by observing the fluorescence of the cooled atomic ions.

The first example concerns the most fundamental molecule having electric dipole-allowed rotational transitions, $\mathrm{HD}^{+}$ $\left({ }^{2} \Sigma_{\mathrm{g}}^{+}\right)$. It consists only of a proton, a deuteron and a single electron. Due to this simple composition, computations from high-level $a b$ initio theory can be compared to high-resolution experiments, helping to determine fundamental constants as the proton mass or the proton-to-electron mass ratio. $\mathrm{HD}^{+}$has an interesting coupling scheme of angular momenta for low values of the rotational angular momentum $N$, in which (in order of the most important energy contributions) first the electron spin $(s=1 / 2)$ and the proton nuclear spin $(I=1 / 2)$ couple, and then the nuclear spin of the deuteron $(I=1)$. The resulting angular momentum of these spins then couples with $N$ to form the total angular momentum $F .^{149}$ Experiments by Schiller and coworkers pursue the spectroscopy of this ion by sympathetic cooling inside a Coulomb crystal of laser-cooled atomic ions $\left(\mathrm{Be}^{+}\right)$, all confined in a linear Paul trap. ${ }^{150-152}$ By this approach, kinetic temperatures as low as approximately $10 \mathrm{mK}$ are achieved for the molecular ions. Additionally, applying a rotational cooling scheme by two IR lasers, the population in $N=0$ may be enhanced compared to the roomtemperature blackbody equilibrium situation. Recently, they achieved to operate the experiment in the Lamb-Dicke regime, in which the Doppler broadening is eliminated. ${ }^{147}$ The LambDicke regime is reached by confining the motion of the species under investigation along the spectroscopy beam direction, $\delta x<\lambda / 2 \pi$. This is most easily achieved for rotational spectroscopy with its long wavelength $\lambda$. They recorded two overlapping hyperfine components $\left(N, F, m_{F}\right)=(1,3, \pm 3) \leftarrow(0,2, \pm 2)$ of the fundamental rotational transition in very high resolution. Here, $m_{F}$ is the projection of $F$ along the quantization axis (a small magnetic field has been applied). The rotational excitation was provided by a multiplier/amplifier chain. The resonant excitation was detected destructively via two following steps, one resonant vibrational overtone transition at $1420 \mathrm{~nm}$ and one non-resonant electronic transition at $266 \mathrm{~nm}$, leading finally to dissociation of $\mathrm{HD}^{+}$. The measurement of the given transition under low-power conditions, as shown in Fig. 18, yields the value $1.3149358280(4)(3) \mathrm{THz}$ (the numbers in parentheses indicating the statistical and systematic error, respectively ${ }^{147}$ ). More recently, the Schiller group improved the described experimental approach, reaching even higher experimental resolution ${ }^{153}$ and enabling the determination of center frequencies with a relative uncertainty on the $10^{-11}$ level. By this, the two Zeeman components included in the signal in Fig. 18 could be resolved. In total, they measured six hyperfine components of the fundamental rotational transition of $\mathrm{HD}^{+}$ (six out of 32 possible), and compared them to high-level $a b$ initio calculations. This comparison yields values for fundamental constants and combinations of them which are able to challenge the current CODATA 2018 set of fundamental constants. For instance, the proton-to-electron mass ratio derived from this experiment has an uncertainty three times smaller
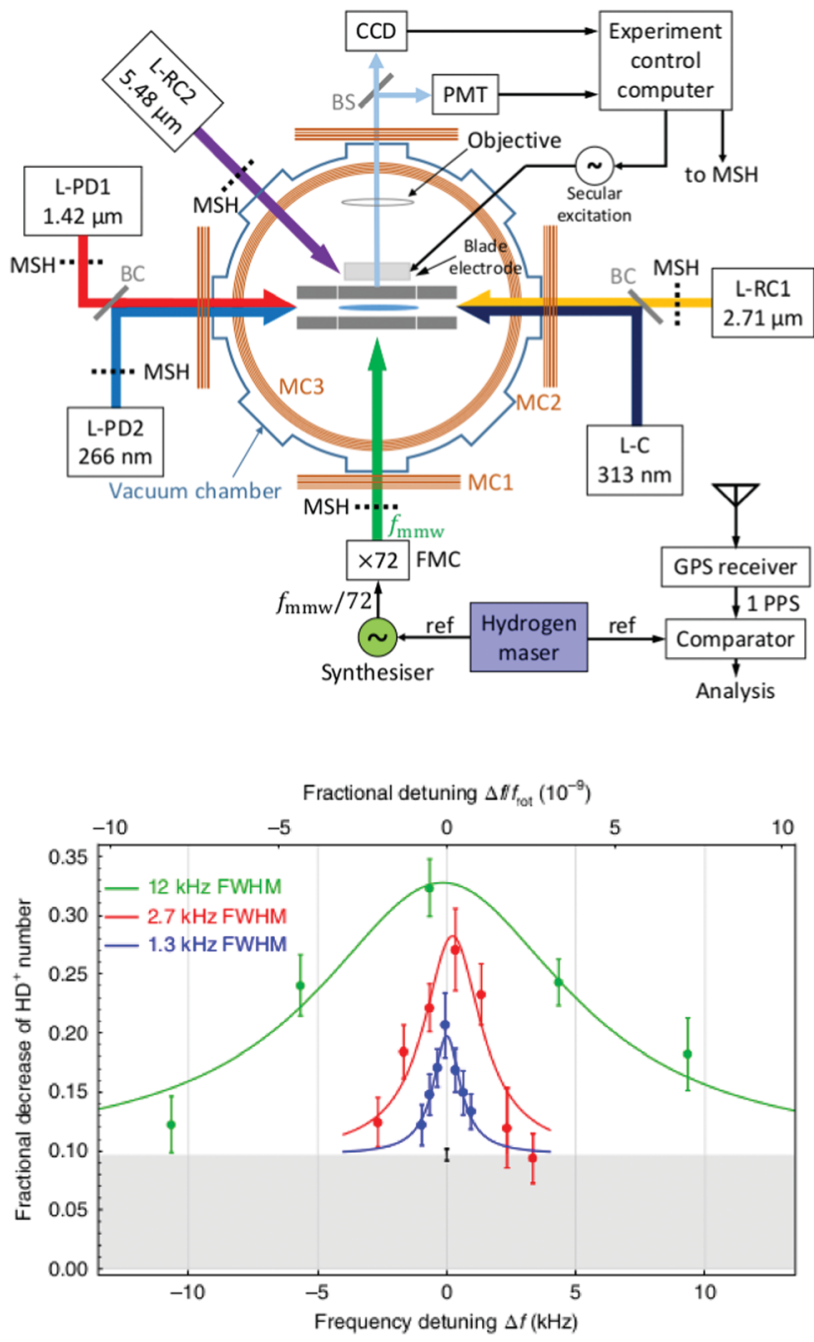

Fig. 18 (Top) Setup for sympathetic cooling and rotational spectroscopy of $\mathrm{HD}^{+}$(L-RC: rotational cooling laser, L-PD: photodissociation laser, L-C: $\mathrm{Be}^{+}$cooling laser). (bottom) Spectroscopy signal of two unresolved hyperfine components $\left(N, F, m_{F}\right)=(1,3, \pm 3) \leftarrow(0,2, \pm 2)$ of the fundamental rotational transition of $\mathrm{HD}^{+}$. The different traces were recorded at different power levels. Figures reprinted from ref. 147 with permission of Springer Nature.

than the value derived from Penning trap experiments. Thus, "for the first time, fundamental constants have been determined with competitive uncertainty making use of the rotational motion of a physical system". ${ }^{153}$

Another very promising approach has been demonstrated very recently for the rotational spectroscopy and quantum state control of a single ${ }^{40} \mathrm{CaH}^{+}$ion. ${ }^{155}$ This ion has a ${ }^{1} \Sigma$ ground state and hyperfine structure due to the nuclear spin $I=1 / 2$ of the proton. The ${ }^{40} \mathrm{CaH}^{+}$ion was co-trapped with a single lasercooled ${ }^{40} \mathrm{Ca}^{+}$ion in the presence of a small magnetic field, leading to the separation of the molecular states with different projection quantum number $m$ (this is the projection of the total angular momentum along the magnetic field, the total angular momentum consisting of rotational angular momentum and the proton nuclear spin). At very low temperature, the 


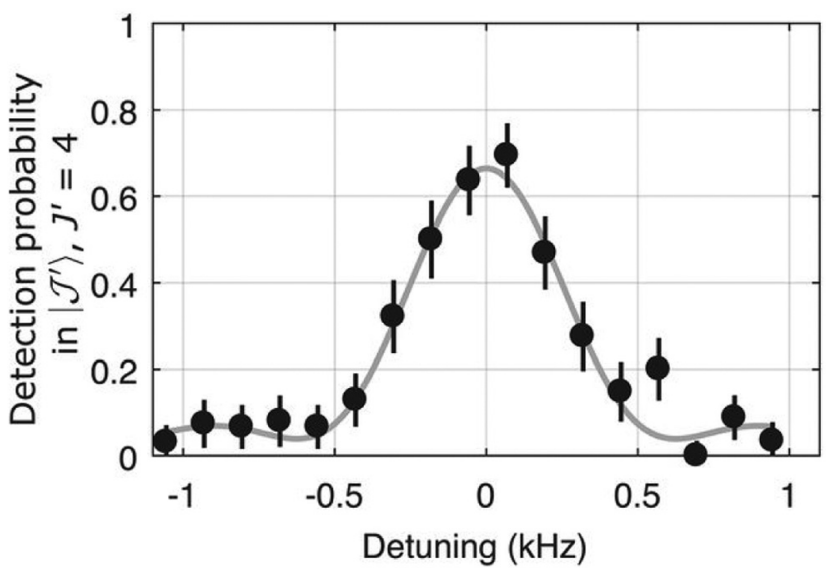

Fig. 19 After preparing a single ${ }^{40} \mathrm{CaH}^{+}$ion in the $(J, m, \xi)=(2,-5 / 2,-)$ state, the probability to be in the $(4,-7 / 2,-)$ state is detected by quantumlogic techniques. ${ }^{154,155}$ When the comb Raman pulses (duration $1.6 \mathrm{~ms}$ ) drive the transition $(4,-7 / 2,-) \leftarrow(2,-5 / 2,-)$ at a difference frequency $1.992911000121(16) \mathrm{THz}$, the ${ }^{40} \mathrm{CaH}^{+}$ion is coherently transferred into this state. Figure reprinted from ref. 155 with permission of AAAS.

motion of both ions is coupled along the longitudinal axis of the ion trap (they occupy harmonic oscillator levels), and the quantum state of ${ }^{40} \mathrm{CaH}^{+}$can be read out non-destructively using quantum logic spectroscopy ${ }^{148,154}$ via the ${ }^{40} \mathrm{Ca}^{+}$atom. Rotational spectroscopy of ${ }^{40} \mathrm{CaH}^{+}$has been done by coherently driving stimulated Raman transitions with two optical frequency combs, which were frequency shifted by acusto-optical modulators to match the allowed Raman transitions with selection rules $\Delta J=0, \pm 2$ and $\Delta m= \pm 1$. Four Raman transitions have been recorded with 11 significant digits, one of which is shown in Fig. 19. Using these four values and ab initio computed corrections to cancel the influence of the magnetic field and the hyperfine structure, the rotational parameters have been determined to fourth order, with a rotational constant $B=142.5017779(17) \mathrm{GHz}$. The beauty of this method lies in the use of highly stable frequency combs operating in the NIR to drive the rotational transitions, without resorting to mm-wave or THz-sources, as well as the high degree of quantum control. When extended to polyatomic molecular ions, in particular chiral ones, many more fundamental discoveries may be expected in the future.

\section{Conclusions and outlook}

The last decade has witnessed the development of many new rotational action spectroscopic schemes. As seen in Section 4, rotational action spectroscopy can be performed by double resonance via a known rovibrational or rovibronic action spectroscopy scheme. This means that if a robust vibrational or electronic action scheme exists for a molecule, the spectroscopy can be readily extended into the rotational domain by such double-resonance methods. This has already been demonstrated for the rotational-LIR (Section 4.2), the rotationalpredissociation (Section 4.3) and the rotational-vibrational LIICG (Section 4.6) double resonance schemes. Novel schemes of this kind are needed to investigate anions (as a complementary tool to non-resonant electron detachment, Section 4.4), or for such ions that eluded the rotational detection by the otherwise general LIICG method (Section 4.5).

Prime targets of the existing action schemes are floppy molecular ions which are best investigated in cryogenic ion traps. Examples include the mentioned hydrogen-helium complexes $\mathrm{H}_{n} \mathrm{He}_{m}{ }^{+},{ }^{27,79,116-118}$ as well as the enigmatic $\mathrm{CH}_{5}{ }^{+23,24,40,156}$ mentioned in the introduction. It is highly desirable to record first mm-wave spectra of $\mathrm{CH}_{5}{ }^{+}$, not only to understand its dynamics, but also for searches of this astrochemically important molecule in space (see e.g. Fig. 1 in ref. 21). The method of choice here is the rotational-vibrational double resonance explained in Section 4.2 using LIR. LIR of $\mathrm{CH}_{5}{ }^{+}$with $\mathrm{CO}_{2}$ is known to have an excellent signal-to-noise ratio and can be operated at low temperature. ${ }^{40,156,157}$ As long as no detailed model of quantum levels and transitions of $\mathrm{CH}_{5}{ }^{+}$ is available ${ }^{158}$ the quest for its $\mathrm{mm}$-wave spectrum has to be done in an unbiased way. For this, one chooses a couple of strong IR transitions out of the 2897 known ones, ${ }^{40}$ and does a double-resonance with a mm-wave source. For a first unbiased search, the mm-wave source is preferably highly tunable, has lower resolution, and high power.

Further technical developments of ion trap machines suitable for rotational action schemes are the extension to temperatures lower than $4 \mathrm{~K}$ (this can be e.g. achieved with pulse tube coolers and will be needed for applying LIICG to anions or for the effective cooling of more complex ions) and the extension to lower excitation frequencies. Concerning the latter point, up to now, rotational action spectroscopy experiments typically have a cutoff frequency limit given by the geometry of the trap electrode orifices which are on the order of $1 \mathrm{~cm}$. The lowestfrequency rotational action spectroscopic measurement up to now was performed at $23 \mathrm{GHz},{ }^{92}$ which already exhibited some broadening artefacts connected with attenuation and excessive use of microwave power. Large and complex molecular ions need access to lower frequencies. This could be accomplished in the future in traps with a long and lateral irradiation window, ${ }^{56}$ or even better, by incorporation of a lateral microwave feed horn into the ion trap. The latter solution would furthermore simplify the setup by replacing a free-space beam by a vacuum-proof RF cable leading to the ion trap.

\section{Conflicts of interest}

There are no conflicts to declare.

\section{Acknowledgements}

The work in Cologne has been supported by the Deutsche Forschungsgemeinschaft (DFG) via SFB 956 (project ID 184018867), sub-project B2, the Gerätezentrum "Cologne Center for Terahertz Spectroscopy” (DFG SCHL 341/15-1), as well as grants AS 319/2-2 and SCHL341/6-1/2. 


\section{Notes and references}

1 S. Weinreb, A. H. Barrett, M. L. Meeks and J. C. Henry, Nature, 1963, 200, 829-831.

2 C. P. Endres, S. Schlemmer, P. Schilke, J. Stutzki and H. S. P. Müller, J. Mol. Spectrosc., 2016, 327, 95-104.

3 H. S. P. Müller, F. Schlöder, J. Stutzki and G. Winnewisser, J. Mol. Struct., 2005, 742, 215-227.

4 T. J. Balle and W. H. Flygare, Rev. Sci. Instrum., 1981, 52, 33-45.

5 G. G. Brown, B. C. Dian, K. O. Douglass, S. M. Geyer, S. T. Shipman and B. H. Pate, Rev. Sci. Instrum., 2008, 79, 053103.

6 R. C. Woods, T. A. Dixon, R. J. Saykally and P. G. Szanto, Phys. Rev. Lett., 1975, 35, 1269-1272.

7 M. Bogey, C. Demuynck, M. Denis, J. L. Destombes and B. Lemoine, Astron. Astrophys., 1984, 137, L15-L16.

8 M. Bogey, H. Bolvin, C. Demuynck and J. L. Destombes, Phys. Rev. Lett., 1987, 58, 988-991.

9 F. C. De Lucia, E. Herbst, G. M. Plummer and G. A. Blake, J. Chem. Phys., 1983, 78, 2312-2316.

10 H. E. Warner, W. T. Conner, R. H. Petrmichl and R. C. Woods, J. Chem. Phys., 1984, 81, 2514.

11 E. Hirota and Y. Endo, J. Mol. Spectrosc., 1988, 127, 527-534.

12 M. C. McCarthy, C. A. Gottlieb, H. Gupta and P. Thaddeus, Astrophys. J., 2006, 652, L141-L144.

13 F. Matsushima, T. Oka and K. Takagi, Phys. Rev. Lett., 1997, 78, 1664-1666.

14 C. A. Gottlieb, A. J. Apponi, M. C. McCarthy, P. Thaddeus and H. Linnartz, J. Chem. Phys., 2000, 113, 1910-1915.

15 G. Cazzoli and C. Puzzarini, J. Chem. Phys., 2005, 123, 041101. 16 T. Amano and T. Hirao, J. Mol. Spectrosc., 2005, 233, 7-14.

17 H. Gupta, S. Brünken, F. Tamassia, C. A. Gottlieb, M. C. McCarthy and P. Thaddeus, Astrophys. J., 2007, 655, L57-L60.

18 T. Amano, Astron. Astrophys., 2010, 516, L4.

19 E. Herbst and W. Klemperer, Astrophys. J., 1973, 185, 505-534.

20 S. Petrie and D. K. Bohme, Mass Spectrom. Rev., 2007, 26, 258-280.

21 M. Larsson, W. D. Geppert and G. Nyman, Rep. Prog. Phys., 2012, 75, 066901.

22 B. A. McGuire, O. Asvany, S. Brünken and S. Schlemmer, Nat. Rev. Phys., 2020, 2, 402-410.

23 E. T. White, J. Tang and T. Oka, Science, 1999, 284, 135-137.

24 T. Oka, Science, 2015, 347, 1313-1314.

25 M. Okumura, L. I. Yeh and Y. T. Lee, J. Chem. Phys., 1985, 83, 3705-3706.

26 L. I. Yeh, M. Okumura, J. D. Myers, J. M. Price and Y. T. Lee, J. Chem. Phys., 1989, 91, 7319-7330.

27 I. Savić, D. Gerlich, O. Asvany, P. Jusko and S. Schlemmer, Mol. Phys., 2015, 113, 2320-2332.

28 M. Töpfer, T. Salomon, S. Schlemmer, O. Dopfer, H. Kohguchi, K. M. T. Yamada and O. Asvany, Phys. Rev. Lett., 2018, 121, 143001.
29 J. Oomens, A. van Roij, G. Meijer and G. von Helden, Astrophys. J., 2000, 542, 404-410.

30 K. R. Asmis, N. L. Pivonka, G. Santambrogio, M. Brümmer, C. Kaposta, D. M. Neumark and L. Wöste, Science, 2003, 299, 1375-1377.

31 N. C. Polfer, Chem. Soc. Rev., 2011, 40, 2211-2221.

32 S. A. Nizkorodov, J. P. Maier and E. J. Bieske, J. Chem. Phys., 1995, 103, 1297-1302.

33 M. Brümmer, C. Kaposta, G. Santambrogio and K. R. Asmis, J. Chem. Phys., 2003, 119, 12700-12703.

34 J. M. Headrick, E. G. Diken, R. S. Walters, N. I. Hammer, R. A. Christie, J. Cui, E. M. Myshakin, M. A. Duncan, M. A. Johnson and K. D. Jordan, Science, 2005, 308, 1765-1769.

35 J. Jašík, J. Žabka, J. Roithová and D. Gerlich, Int. J. Mass Spectrom., 2013, 354, 204-210.

36 E. K. Campbell, M. Holz, D. Gerlich and J. P. Maier, Nature, 2015, 523, 322-323.

37 S. Schlemmer, T. Kuhn, E. Lescop and D. Gerlich, Int. J. Mass Spectrom., 1999, 185, 589-602.

38 S. Schlemmer, E. Lescop, J. V. Richthofen and D. Gerlich, J. Chem. Phys., 2002, 117, 2068-2075.

39 O. Asvany, T. Giesen, B. Redlich and S. Schlemmer, Phys. Rev. Lett., 2005, 94, 073001.

40 O. Asvany, K. M. T. Yamada, S. Brünken, A. Potapov and S. Schlemmer, Science, 2015, 347, 1346-1349.

41 S. Chakrabarty, M. Holz, E. K. Campbell, A. Banerjee, D. Gerlich and J. P. Maier, J. Phys. Chem. Lett., 2013, 4, 4051-4054.

42 O. Asvany, S. Brünken, L. Kluge and S. Schlemmer, Appl. Phys. B, 2014, 114, 203-211.

43 H. Kohguchi, P. Jusko, K. M. T. Yamada, S. Schlemmer and O. Asvany, J. Chem. Phys., 2018, 148, 144303.

44 J. L. Doménech, P. Jusko, S. Schlemmer and O. Asvany, Astrophys. J., 2018, 857, 61.

45 O. Asvany, O. Ricken, H. S. P. Müller, M. C. Wiedner, T. Giesen and S. Schlemmer, Phys. Rev. Lett., 2008, 100, 233004.

46 A. Svendsen, U. J. Lorenz, O. V. Boyarkin and T. R. Rizzo, Rev. Sci. Instrum., 2010, 81, 073107.

47 J. G. Redwine, Z. A. Davis, N. L. Burke, R. A. Oglesbee, S. A. McLuckey and T. S. Zwier, Int. J. Mass Spectrom., 2013, 348, 9-14.

48 N. Heine and K. R. Asmis, Int. Rev. Phys. Chem., 2015, 34, 1-34.

49 S. Xu, S. Gozem, A. I. Krylov, C. R. Christopher and J. Mathias Weber, Phys. Chem. Chem. Phys., 2015, 17, 31938-31946.

50 A. Günther, P. Nieto, D. Müller, A. Sheldrick, D. Gerlich and O. Dopfer, J. Mol. Spectrosc., 2017, 332, 8-15.

51 S. Spieler, C. H. Duong, A. Kaiser, F. Duensing, K. Geistlinger, M. Fischer, N. Yang, S. S. Kumar, M. A. Johnson and R. Wester, J. Phys. Chem. A, 2018, 122, 8037-8046.

52 P. Jusko, S. Brünken, O. Asvany, S. Thorwirth, A. Stoffels, L. van der Meer, G. Berden, B. Redlich, J. Oomens and S. Schlemmer, Faraday Discuss., 2019, 217, 172-202. 
53 E. K. Campbell and P. W. Dunk, Rev. Sci. Instrum., 2019, 90, 103101.

54 D. Gerlich, Phys. Scr., 1995, T59, 256-263.

55 O. Asvany, F. Bielau, D. Moratschke, J. Krause and S. Schlemmer, Rev. Sci. Instr., 2010, 81, 076102.

56 K. Geistlinger, M. Fischer, S. Spieler, L. Remmers, F. Duensing, F. Dahlmann, E. Endres and R. Wester, Rev. Sci. Instr., 2021, 92, 023204.

57 N. R. Daly, Rev. Sci. Instr., 1960, 31, 264-267.

58 W. H. Wing, G. A. Ruff, W. E. Lamb and J. J. Spezeski, Phys. Rev. Lett., 1976, 36, 1488-1491.

59 D. E. Tolliver, G. A. Kyrala and W. H. Wing, Phys. Rev. Lett., 1979, 43, 1719-1722.

60 J. T. Shy, J. W. Farley, W. E. Lamb and W. H. Wing, Phys. Rev. Lett., 1980, 45, 535-537.

61 J.-T. Shy, J. W. Farley and W. H. Wing, Phys. Rev. A: At., Mol., Opt. Phys., 1981, 24, 1146-1149.

62 M. Okumura, L. I. Yeh and Y. T. Lee, J. Chem. Phys., 1988, 88, 79-91.

63 D. W. Boo, Z. Liu, A. G. Suits, J. Tse and Y. T. Lee, Science, 1995, 269, 57-59.

64 D. W. Boo and Y. T. Lee, J. Chem. Phys., 1995, 103, 520-530.

65 R. V. Olkhov, S. A. Nizkorodov and O. Dopfer, J. Chem. Phys., 1999, 110, 9527-9535.

66 S. A. Nizkorodov, D. Roth, R. V. Olkhov, J. P. Maier and O. Dopfer, Chem. Phys. Lett., 1997, 278, 26-30.

67 E. Bieske and O. Dopfer, Chem. Rev., 2000, 100, 3963-3998. 68 O. Dopfer, Int. Rev. Phys. Chem., 2003, 22, 437-495.

69 J. Jašík, R. Navrátil, I. Němec and J. Roithová, J. Phys. Chem. A, 2015, 119, 12648-12655.

70 O. V. Boyarkin, S. R. Mercier, A. Kamariotis and T. R. Rizzo, J. Am. Chem. Soc., 2006, 128, 2816-2817.

71 M.-F. Jagod, M. Rösslein, C. M. Gabrys, B. D. Rehfuss, F. Scappini, M. W. Crofton and T. Oka, J. Chem. Phys., 1992, 97, 7111-7123.

72 S. Schlemmer, O. Asvany and T. Giesen, Phys. Chem. Chem. Phys., 2005, 7, 1592-1600.

73 A. Patzer, M. Schütz, T. Möller and O. Dopfer, Angew. Chem., Int. Ed., 2012, 51, 4925-4929.

74 A. G. Császár, T. Szidarovszky, O. Asvany and S. Schlemmer, Mol. Phys., 2019, 117, 1559.

75 P. Jusko, C. Konietzko, S. Schlemmer and O. Asvany, J. Mol. Spectrosc., 2016, 319, 55-58.

76 P. Jusko, A. Stoffels, S. Thorwirth, S. Brünken, S. Schlemmer and O. Asvany, J. Mol. Spectrosc., 2017, 332, 59-66.

77 C. Markus, S. Thorwirth, O. Asvany and S. Schlemmer, Phys. Chem. Chem. Phys., 2019, 21, 26406-26412.

78 J. L. Doménech, O. Asvany, C. R. Markus, S. Schlemmer and S. Thorwirth, J. Mol. Spectrosc., 2020, 374, 111375.

79 M. Töpfer, A. Jensen, K. N. Anders, H. Kohguchi, T. Szidarovszky, A. G. Császár, S. Schlemmer and O. Asvany, Phys. Chem. Chem. Phys., 2020, 22, 22885.

80 S. Brünken, L. Kluge, A. Stoffels, J. Pérez-Ríos and S. Schlemmer, J. Mol. Spectrosc., 2017, 332, 67-78.
81 D. A. Jennings, C. Demuynck, M. Banek and K. M. Evenson, unpublished.

82 J. Ramanlal and J. Tennyson, Mon. Not. R. Astron. Soc., 2004, 354, 161-168.

83 S. Schlemmer, O. Asvany, E. Hugo and D. Gerlich, IAU Symposium 231, 2005, pp. 125-134.

84 D. Oepts, A. F. G. van der Meer and P. W. van Amersfoort, Infrared Phys. Technol., 1995, 36, 297-308.

85 S. C. Foster, A. R. W. McKellar, I. R. Peterkin, J. K. G. Watson, F. S. Pan, M. W. Crofton, R. S. Altman and T. Oka, J. Chem. Phys., 1986, 84, 91-99.

86 T. Yonezu, F. Matsushima, Y. Moriwaki, K. Takagi and T. Amano, J. Mol. Spectrosc., 2009, 256, 238-241.

87 T. Amano, Philos. Trans. R. Soc., A, 2006, 364, 2943-2952.

88 S. Brünken, O. Sipilä, E. T. Chambers, J. Harju, P. Caselli, O. Asvany, C. E. Honingh, T. Kamiński, K. M. Menten, J. Stutzki and S. Schlemmer, Nature, 2014, 516, 219-221.

89 J. Harju, O. Sipila, S. Brňnken, S. Schlemmer, P. Caselli, M. Juvela, K. M. Menten, J. Stutzki, O. Asvany, T. Kamiński, Y. Okada and R. Higgins, Astrophys. J., 2017, 840, 63.

90 H. Roberts, E. Herbst and T. J. Millar, Mon. Not. R. Astron. Soc., 2002, 336, 283-290.

91 D. Smith, N. Adams and E. Alge, Astrophys. J., 1982, 263, 123-129.

92 M. Töpfer, P. Jusko, S. Schlemmer and O. Asvany, Astron. Astrophys., 2016, 593, L11.

93 R. H. Page, Y. R. Shen and Y. T. Lee, J. Chem. Phys., 1988, 88, 5362-5376.

94 M. Guidi, U. J. Lorenz, G. Papadopoulos, O. V. Boyarkin and T. R. Rizzo, J. Phys. Chem. A, 2009, 113, 797-799.

95 S. Gärtner, J. Krieg, A. Klemann, O. Asvany, S. Brünken and S. Schlemmer, J. Phys. Chem. A, 2013, 117, 9975-9984.

96 P. Jusko, M. Töpfer, H. S. P. Müller, P. N. Ghosh, S. Schlemmer and O. Asvany, J. Mol. Spectrosc., 2017, 332, 33-37.

97 O. Asvany, E. Hugo, F. Müller, F. Kühnemann, S. Schiller, J. Tennyson and S. Schlemmer, J. Chem. Phys., 2007, 127, 154317.

98 T. Amano and J. K. G. Watson, J. Chem. Phys., 1984, 81, 2869-2871.

99 P. Jusko, O. Asvany, A.-C. Wallerstein, S. Brünken and S. Schlemmer, Phys. Rev. Lett., 2014, 112, 253005.

100 F. Matsushima, T. Yonezu, T. Okabe, K. Tomaru and Y. Moriwaki, J. Mol. Spectrosc., 2006, 235, 261-264.

101 O. Asvany, S. Thorwirth, B. Redlich and S. Schlemmer, J. Mol. Spectrosc., 2018, 347, 1-6.

102 M. Rösslein, M. F. Jagod, C. M. Gabrys and T. Oka, Astrophys. J., 1991, 382, L51-L53.

103 Y.-S. Wang, C.-H. Tsai, Y. T. Lee, H.-C. Chang, J. C. Jiang, O. Asvany, S. Schlemmer and D. Gerlich, J. Phys. Chem. A, 2003, 107, 4217-4225.

104 S. A. Nizkorodov, M. Meuwly, J. P. Maier, O. Dopfer and E. J. Bieske, J. Chem. Phys., 1998, 108, 8964-8975.

105 R. V. Olkhov, S. A. Nizkorodov and O. Dopfer, J. Chem. Phys., 1998, 108, 10046-10060. 
106 O. Dopfer, R. V. Olkhov and J. P. Maier, J. Chem. Phys, 2000, 112, 2176-2186.

107 O. Dopfer, N. Solcá, R. V. Olkhov and J. P. Maier, Chem. Phys., 2002, 283, 85-110.

108 D. C. McDonald, D. T. Mauney, D. Leicht, J. H. Marks, J. A. Tan, J.-L. Kuo and M. A. Duncan, J. Chem. Phys., 2016, 145, 231101.

109 T. Salomon, M. Töpfer, P. Schreier, S. Schlemmer, H. Kohguchi, L. Surin and O. Asvany, Phys. Chem. Chem. Phys., 2018, 21, 3440.

110 E. Bieske, S. Nizkorodov, F. Bennett and J. Maier, J. Chem. Phys., 1995, 102, 5152-5164.

111 S. A. Nizkorodov, J. P. Maier and E. J. Bieske, J. Chem. Phys., 1995, 102, 5570-5571.

112 O. Dopfer, D. Roth and J. P. Maier, Chem. Phys. Lett., 1999, 310, 201-208.

113 E. Bieske, A. Soliva, A. Friedmann and J. Maier, J. Chem. Phys., 1992, 96, 4035-4036.

114 T. Salomon, J. L. Doménech, P. C. Schmid, E. A. Michael, S. Schlemmer and O. Asvany, J. Mol. Spectrosc., 2021, 377, 111421.

115 D. Papp, A. G. Császár, K. Yamanouchi and T. Szidarovszky, J. Chem. Theory Comput., 2018, 14, 1523-1533.

116 O. Asvany, S. Schlemmer, A. van der Avoird, T. Szidarovszky and A. G. Császár, J. Mol. Spectrosc., 2021, 377, 111423.

117 O. Asvany, S. Schlemmer, T. Szidarovszky and A. G. Császár, J. Phys. Chem. Lett., 2019, 10, 5325-5330.

118 A. Carrington, D. I. Gammie, A. M. Shaw, S. M. Taylor and J. M. Hutson, Chem. Phys. Lett., 1996, 260, 395-405.

119 D. I. Gammie, J. C. Page and A. M. Shaw, J. Chem. Phys., 2002, 116, 6072-6078.

120 D. Koner, J. C. San Vicente Veliz, A. van der Avoird and M. Meuwly, Phys. Chem. Chem. Phys., 2019, 21, 24976-24983.

121 S. Brünken, H. Gupta, C. A. Gottlieb, M. C. McCarthy and P. Thaddeus, Astrophys. J., 2007, 664, L43-L46.

122 R. Otto, A. von Zastrow, T. Best and R. Wester, Phys. Chem. Chem. Phys., 2013, 15, 612-618.

123 S. Lee, D. Hauser, O. Lakhmanskaya, S. Spieler, E. S. Endres, K. Geistlinger, S. S. Kumar and R. Wester, Phys. Rev. A, 2016, 93, 032513.

124 F. Goldfarb, C. Drag, W. Chaibi, S. Kröger, C. Blondel and C. Delsart, J. Chem. Phys., 2005, 122, 014308.

125 G. Cazzoli and C. Puzzarini, Astrophys. J., Lett., 2006, 648, L79-L81.

126 C. M. Persson, M. Hajigholi, G. E. Hassel, A. O. H. Olofsson, J. H. Black, E. Herbst, H. S. P. Müller, J. Cernicharo, E. S. Wirström, M. Olberg, Å. Hjalmarson, D. C. Lis, H. M. Cuppen, M. Gerin and K. M. Menten, Astron. Astrophys., 2014, 567, A130.

127 O. Lakhmanskaya, M. Simpson, S. Murauer, M. Nötzold, E. Endres, V. Kokoouline and R. Wester, Phys. Rev. Lett., 2018, 120, 253003.

128 O. Lakhmanskaya, M. Simpson, S. Murauer, V. Kokoouline and R. Wester, J. Chem. Phys., 2018, 149, 104302.
129 M. Okumura, L. I. Yeh, J. D. Myers and Y. T. Lee, J. Phys. Chem., 1990, 94, 3416-3427.

130 A. M. Ricks, G. E. Douberly, P. V. R. Schleyer and M. A. Duncan, J. Chem. Phys., 2010, 132, 051101.

131 M. Z. Kamrath, R. A. Relph, T. L. Guasco, C. M. Leavitt and M. A. Johnson, Int. J. Mass Spectrom., 2011, 300, 91-98.

132 E. J. Bieske, A. Soliva, M. A. Welker and J. P. Maier, J. Chem. Phys., 1990, 93, 4477-4478.

133 S. Brünken, L. Kluge, A. Stoffels, O. Asvany and S. Schlemmer, Astrophys. J., Lett., 2014, 783, L4.

134 S. Yu, B. J. Drouin, J. C. Pearson and T. Amano, J. Mol. Spectrosc., 2018, 350, 30-36.

135 T. Amano, Astrophys. J., Lett., 2010, 716, L1-L3.

136 J. Pety, P. Gratier, V. Guzmán, E. Roueff, M. Gerin, J. R. Goicoechea, S. Bardeau, A. Sievers, F. Le Petit, J. Le Bourlot, A. Belloche and D. Talbi, Astron. Astrophys., 2012, 548, A68.

137 B. A. McGuire, P. B. Carroll, R. A. Loomis, G. A. Blake, J. M. Hollis, F. J. Lovas, P. R. Jewell and A. J. Remijan, Astrophys. J., 2013, 774, 56.

138 A. Stoffels, L. Kluge, S. Schlemmer and S. Brünken, Astron. Astrophys., 2016, 593, A56.

139 J. L. Doménech, S. Schlemmer and O. Asvany, Astrophys. J., 2017, 849, 60.

140 J. L. Doménech, S. Schlemmer and O. Asvany, Astrophys. J., 2018, 866, 158.

141 S. Thorwirth, P. Schreier, T. Salomon, S. Schlemmer and O. Asvany, Astrophys. J. Lett., 2019, 882, L6.

142 P. C. Schmid, S. Thorwirth, C. P. Endres, M. Töpfer, Á. Sánchez-Monge, A. Schwörer, P. Schilke, S. Schlemmer and O. Asvany, Front. Astron. Space Sci., 2021, submitted.

143 O. Asvany, C. R. Markus, A. Roucou, S. Schlemmer, S. Thorwirth and C. Lauzin, J. Mol. Spectrosc., 2021, 378, 111447.

144 O. Asvany, C. R. Markus, K. Nagamori, H. Kohguchi, J. Furuta, K. Kobayashi, P. C. Schmid, S. Schlemmer and S. Thorwirth, Astrophys. J., 2021, 910, 15.

145 F. Grandinetti, Int. J. Mass Spectrom., 2004, 237, 243-267.

146 O. Asvany, C. R. Markus, T. Salomon, P. C. Schmid, S. Banhatti, S. Brünken, F. Lipparini, J. Gauss and S. Schlemmer, J. Mol. Struct., 2020, 1214, 128023.

147 S. Alighanbari, M. G. Hansen, V. I. Korobov and S. Schiller, Nat. Phys., 2018, 14, 555-559.

148 P. O. Schmidt, T. Rosenband, C. Langer, W. M. Itano, J. C. Bergquist and D. J. Wineland, Science, 2005, 309, 749-752.

149 D. Bakalov, V. I. Korobov and S. Schiller, Phys. Rev. Lett., 2006, 97, 243001.

150 B. Roth, J. C. J. Koelemeij, H. Daerr and S. Schiller, Phys. Rev. A: At., Mol., Opt. Phys., 2006, 74, 040501.

151 J. Shen, A. Borodin, M. Hansen and S. Schiller, Phys. Rev. A: At., Mol., Opt. Phys., 2012, 85, 032519.

152 I. V. Kortunov, S. Alighanbari, M. G. Hansen, G. S. Giri, V. I. Korobov and S. Schiller, Nat. Phys., 2021, 17, 569-573.

153 S. Alighanbari, G. S. Giri, F. L. Constantin, V. I. Korobov and S. Schiller, Nature, 2020, 581, 152-158. 
154 C.-W. Chou, C. Kurz, D. B. Hume, P. N. Plessow, D. R. Leibrandt and D. Leibfried, Nature, 2017, 545, 203-207.

155 C. W. Chou, A. L. Collopy, C. Kurz, Y. Lin, M. E. Harding, P. N. Plessow, T. Fortier, S. Diddams, D. Leibfried and D. R. Leibrandt, Science, 2020, 367, 1458-1461.
156 O. Asvany, P. Kumar, P. B. Redlich, I. Hegemann, S. Schlemmer and D. Marx, Science, 2005, 309, 1219-1222. 157 O. Asvany, J. Krieg and S. Schlemmer, Rev. Sci. Instr., 2012, 83, 093110.

158 H. Schmiedt, P. Jensen and S. Schlemmer, Phys. Rev. Lett., 2016, 117, 223002. 